\title{
On 2D elliptic discontinuous Galerkin methods
}

\author{
S. J. Sherwin ${ }^{1, *, \dagger}$, R. M. Kirby ${ }^{2,}$, J. Peiró ${ }^{1, \S}$, \\ R. L. Taylor ${ }^{3, \mathbb{I}, \|}$ and O. C. Zienkiewicz ${ }^{4, * *}$ \\ ${ }^{1}$ Department of Aeronautics, Imperial College London, U.K. \\ ${ }^{2}$ School of Computing, University of Utah, U.S.A. \\ ${ }^{3}$ Department of Civil and Environmental Engineering, University of California at Berkeley, U.S.A. \\ ${ }^{4}$ Department of Civil Engineering, University of Wales, Swansea, U.K.
}

\begin{abstract}
SUMMARY
We discuss the discretization using discontinuous Galerkin (DG) formulation of an elliptic Poisson problem. Two commonly used DG schemes are investigated: the original average flux proposed by Bassi and Rebay (J. Comput. Phys. 1997; 131:267) and the local discontinuous Galerkin (LDG) (SIAM J. Numer. Anal. 1998; 35:2440-2463) scheme. In this paper we expand on previous expositions (Discontinuous Galerkin Methods: Theory, Computation and Applications. Springer: Berlin, 2000; 135-146; SIAM J. Sci. Comput. 2002; 24(2):524-547; Int. J. Numer. Meth. Engng. 2003; 58(2): 1119-1148) by adopting a matrix based notation with a view to highlighting the steps required in a numerical implementation of the DG method. Through consideration of standard $C^{0}$-type expansion bases, as opposed to elementally orthogonal expansions, with the matrix formulation we are able to apply static condensation techniques to improve efficiency of the direct solver when high order expansions are adopted. The use of $C^{0}$-type expansions also permits the direct enforcement of Dirichlet boundary conditions through a 'lifting' approach where the LDG flux does not require further stabilization. In our construction we also adopt a formulation of the continuous DG fluxes that permits a more general interpretation of their numerical implementation. In particular it allows us to determine the conditions under which the LDG method provides a near local stencil. Finally a study of the conditioning and the size of the null space of the matrix systems resulting from the DG discretization of the elliptic problem is undertaken. Copyright (c) 2005 John Wiley \& Sons, Ltd.
\end{abstract}

KEY WORDS: discontinuous Galerkin; spectral element; hp finite element; elliptic problems

\footnotetext{
*Correspondence to: S. J. Sherwin, Department of Aeronautics, Imperial College London, South Kensington Campus, London, SW7 2AZ

†E-mail: s.sherwin@imperial.ac.uk

*E-mail: kirby@cs.utah.edu

§E-mail: j.peiro@imperial.ac.uk

IE-mail: rlt@ce.vulture.berkeley.edu

$\|$ Visiting Professor, CIMNE, UPC, Barcelona, Spain.

**Unesco Professor, CIMNE, UPC, Barcelona, Spain.
}

Contract/grant sponsor: Royal Academy of Engineering

Contract/grant sponsor: NSF; Contract/grant number: NSF-CCF 0347791

Copyright (c) 2005 John Wiley \& Sons, Ltd.

Received 14 February 2005

Revised 9 June 2005

Accepted 22 July 2005 


\section{INTRODUCTION}

Although the original thrust of most discontinuous Galerkin research was the solution of hyperbolic problems, the general proliferation of the DG methodology has also spread to the study of parabolic and elliptic problems. For example, works such as Reference [1], in which the viscous compressible Navier-Stokes equations were solved, required that a discontinuous Galerkin formulation be extended beyond the hyperbolic advection terms to the viscous terms of the Navier-Stokes equations. Concurrently, other discontinuous Galerkin formulations for parabolic and elliptic problems were proposed [2-7].

In an effort to classify existing DG methods for elliptic problems, Arnold et al. published, first in Reference [8] and then more fully in Reference [9], a unified analysis of discontinuous Galerkin methods for elliptic problems. There have subsequently been several attempts to provide performance information concerning the choice of continuous fluxes used in these methods, both by the developers of different flux choices (e.g. References [2-7]) and by those interested in flux choice comparisons and analysis (e.g. References [10-15]). For an overview of many of the properties of the discontinuous Galerkin method, from the theoretical, performance and application perspectives we refer the reader to the review article [16] and the references therein.

Following the formulation of Arnold et al. [9], the second-order elliptic DG matrix systems can be recast in terms of a larger first-order system through the introduction of an auxiliary variable. Ultimately the system can be recombined to obtain the so-called primal form of problem. Since this approach fits very naturally into the way the DG formulation is applied to first-order hyperbolic problems, we will follow this construction in our exposition. In doing so we still need to define a single valued flux at the elemental interfaces which in turn determines the type of DG formulation. We will concentrate on two of the more commonly used formulations currently being adopted from the set of 'numerical fluxes independent of $\nabla u_{h}$ ' [9], namely: Bassi-Rebay [1] and Local Discontinuous Galerkin (LDG) [4]. Specific details regarding the theoretical foundations for the multi-dimensional LDG have been presented in References $[14,17,18]$. We also note that the LDG has been successfully applied in the solution of non-trivial elliptic problems such as the Stokes system [19] and the Oseen equations [20].

Building upon Reference [21], the primary motivation behind this paper is to illustrate how to efficiently implement the multi-dimensional DG schemes and to compare the formulation with the standard continuous Galerkin implementation. We have addressed the following issues in our investigations of DG methods for elliptic problems:

- Whilst the paper by Arnold et al. [9] is very comprehensive and rigorous, it is directed towards the mathematical understanding rather than the numerical implementation. For example, their definition of the continuous elemental fluxes for a component of the auxiliary variables is based on an average of elemental contributions of all components of the auxiliary variables coupled through the edge normal. In Sections 3.2.3 and 3.2.4 we introduce an equivalent numerical flux definition where the flux for a component of the auxiliary variable is only dependent upon geometric information and the same component of the auxiliary variable and so is amenable to matrix implementation.

- The DG formulation permits elementally discontinuous expansions to be adopted and so we can consider using elementally orthogonal expansions which are numerically attractive since elemental mass matrices are diagonal. However, the use of polynomial expansions 
with boundary-interior decompositions, designed to enforce $C^{0}$ continuity in continuous Galerkin methods, provide the following numerical benefits.

- Unlike the elemental orthogonal expansion, the $C^{0}$-type expansions are amenable to the application of static condensation techniques which lead to Schur complement matrix systems with improved conditioning, particularly for higher order polynomial approximations.

- When using an orthogonal basis, the common practice is to use a penalty method to impose Dirichlet boundary conditions. When applying a $C^{0}$-type basis with a boundary-interior decomposition Dirichlet conditions can be directly imposed through global lifting (or homogenization) as is commonly applied in continuous Galerkin methods.

- Finally, we have observed that stabilization is not required in an LDG scheme when Dirichlet boundary conditions are directly enforced through a lifting type operation applicable when using $C^{0}$-type expansion.

The paper is organized as follows: Section 2 provides a summary of the notation adopted throughout the paper. Section 3 presents a full derivation of the discontinuous Galerkin formulation applied to the elliptic diffusion operator with a variable diffusivity tensor. This presentation starts from the continuous formulation to introduce topics such as stabilization, flux averaging for the Bassi-Rebay and local discontinuous Galerkin (LDG) formulations and boundary condition enforcement. After the introduction of the continuous problem we formulate in Section 3.3 the discretization in terms of a matrix representation which is more amenable to a numerical implementation of these schemes. In Section 3.4 we discuss different elemental polynomial expansions that can be applied in the DG formulations. This allows us to consider how the static condensation technique can be applied in a DG formulation in Section 3.5. In Section 4 we analyse the increased null space dimension of the DG formulation and associated conditioning. Finally in Section 5 we discuss some numerical solutions of smooth and non-smooth elliptic problems.

\section{NOTATION}

The following notation will be adopted in this paper.

\subsection{Regions}

$\begin{array}{ll}\mathbf{x} & \text { Cartesian co-ordinates, } \mathbf{x}=\left[x_{1}, x_{2}\right]^{\mathrm{T}} \\ \Omega & \text { global computational domain } \\ \partial \Omega & \text { boundary of computational domain } \Omega \\ \partial \Omega_{\mathrm{D}} & \text { boundary of computational domain } \Omega \text { with Dirichlet boundary conditions } \\ \partial \Omega_{\mathrm{N}} & \text { boundary of computational domain } \Omega \text { with Neumann boundary conditions } \\ \Omega^{e} & \text { elemental region } e \text { in } \Omega, \Omega=\bigcup_{e=1}^{N_{\mathrm{el}}} \Omega^{e} \\ \partial \Omega^{e} & \text { boundary of element } e \\ \partial \Omega_{i}^{e} & i \text { th boundary segment of boundary } \partial \Omega^{e}, \text { where } \partial \Omega^{e}=\bigcup_{i=1}^{N_{b}^{e}} \partial \Omega_{i}^{e} \text { and } \partial \Omega_{i}^{e} \cap \partial \Omega_{j}^{e}=\emptyset \\ \mathbf{n}^{e} & \text { when } i \neq j\end{array}$




\subsection{Variables}

$u^{e}(\mathbf{x}) \quad$ primary solution variable in element $e$

$q_{i}^{e}(\mathbf{x}) \quad$ auxiliary solution variable in element $e$, i.e. $q_{i}^{e}=\partial u^{e} / \partial x_{i}$

$\mathbf{q}^{e} \quad$ vector of auxiliary functions on an element $\Omega^{e}$, i.e. $\mathbf{q}^{e}=\left[q_{1}^{e}, q_{2}^{e}, q_{3}^{e}\right]^{\mathrm{T}}$

$\tilde{\mathbf{q}}^{e} \quad$ vector of auxiliary functions on an element $\Omega^{e}$ which are continuous over adjacent elements

$\mathscr{D} \quad$ diffusivity tensor $\mathscr{D}[i, j]=\mathscr{D}_{i j}, 1 \leqslant i, j \leqslant 3$

\subsection{Integers}

$N_{\mathrm{el}} \quad$ number of elemental regions

$N_{b}^{e} \quad$ number of boundary segments (or faces) in element $e$

$N_{q}^{e} \quad$ number of elemental degrees of freedom in auxiliary variable $q^{e}(\mathbf{x})$

$N_{u}^{e} \quad$ number of elemental degrees of freedom in primary variable $u^{e}(\mathbf{x})$

\subsection{Inner products}

$(u, v)_{\Omega^{e}}$ inner product of the scalar functions $u(\mathbf{x})$ and $v(\mathbf{x})$ over element $e$, i.e. $\int_{\Omega^{e}} u(\mathbf{x}) v(\mathbf{x}) \mathrm{d} \mathbf{x}$

$(\mathbf{a}, \mathbf{b})_{\Omega^{e}} \quad$ inner product of two vector functions $\mathbf{a}(\mathbf{x})$ and $\mathbf{b}(\mathbf{x})$ over element $e$, i.e. $\int_{\Omega^{e}} \mathbf{a}(\mathbf{x})$. $\mathbf{b}(\mathbf{x}) \mathrm{d} \mathbf{x}$

$\langle u, v\rangle_{\partial \Omega^{e}}$ inner product along the boundary $\partial \Omega^{e}$ of element $e$, i.e. $\langle u, v\rangle_{\partial \Omega^{e}}=\int_{\partial \Omega^{e}} u(s) v(s) \mathrm{d} s$ $=\sum_{i=1}^{N_{b}^{e}}\langle u, v\rangle_{\partial \Omega_{i}^{e}}$

$\langle u, v\rangle_{\partial \Omega_{i}^{e}}$ inner product along the $i$ th edge (face) of element boundary $\partial \Omega^{e}$

\subsection{Discrete matrices and vectors}

$\phi_{j}^{e}(\mathbf{x}) \quad j$ th expansion basis in element $\Omega^{e} ; j=1, \ldots, N_{u}^{e}$ (or $N_{q}^{e}$ depending on variable).

$\underline{\hat{u}}^{e}[j] \quad j$ th expansion coefficients for the primitive variable in element $\Omega^{e}$ such that $u^{e}(\mathbf{x})=\sum_{j=1}^{N_{u}^{e}} \underline{\hat{u}}^{e}[j] \phi_{j}(\mathbf{x})$

$\underline{\hat{q}}_{i}^{e}[j] \quad j$ th expansion coefficients for the $i$ th auxiliary variable in element $\Omega^{e}$ such that $q_{i}^{e}(\mathbf{x})=\sum_{j=1}^{N_{q}^{e}} \underline{\hat{q}}_{i}^{e}[j] \phi_{j}(\mathbf{x})$

$\mathbb{M}^{e} \quad$ elemental mass matrix, i.e. $\mathbb{M}^{e}[i, j]=\left(\phi_{i}, \phi_{j}\right)_{\Omega^{e}}$

$\mathbb{D}_{k}^{e} \quad$ elemental weak derivative matrix of the expansion basis with respect to the $x_{k}$ direction, i.e. $\mathbb{D}_{k}^{e}[i, j]=\left(\phi_{i}, \frac{\partial \phi_{j}}{\partial x_{k}}\right)_{\Omega^{e}}$

$\hat{\mathbb{D}}_{k}^{e} \quad$ elemental weak matrix of the $k$ th component of the gradient of the expansion basis multiplied by diffusivity tensor, i.e. $\hat{\mathbb{D}}_{k}^{e}[i, j]=\left(\phi_{i}^{e}, \mathscr{D}_{k 1} \frac{\partial}{\partial x_{1}} \phi_{j}^{e}+\mathscr{D}_{k 2} \frac{\partial}{\partial x_{2}} \phi_{j}^{e}\right)_{\Omega^{e}}$

$\tilde{\mathbb{D}}_{k}^{e} \quad$ adjoint operator of $\hat{\mathbb{D}}_{k}^{e}[i, j]$, e.g. $\tilde{\mathbb{D}}_{k}^{e}[i, j]=\left(\phi_{i}^{e}, \frac{\partial}{\partial x_{1}}\left[\mathscr{D}_{1 k} \phi_{j}^{e}\right]+\frac{\partial}{\partial x_{2}}\left[\mathscr{D}_{2 k} \phi_{j}^{e}\right]\right)_{\Omega^{e}}$

$\mathbb{E}_{k l}^{e, f} \quad$ elemental matrix of the inner product over $\partial \Omega_{l}^{e}$ (edge $l$ of element $e$ ) of basis $\phi_{i}^{e}$ in element $e$ with basis $\phi_{j}^{f}$ of element $f$ weighted with the $k$ th component of $\mathbf{n}^{e}$, i.e. $\mathbb{E}_{k l}^{e, f}[i, j]=\left\langle\phi_{i}^{e}, \phi_{j}^{f} n_{k}^{e}\right\rangle_{\partial \Omega_{l}^{e}}$ 
$\mathbb{F}_{k l}^{e, f} \quad$ elemental matrix (for the $k$ th flux) of the inner product over $\partial \Omega_{l}^{e}$ such that $\mathbb{F}_{k l}^{e, f}[i, j]=\left\langle\left(n_{1}^{e} \mathscr{D}_{1 k}+n_{2}^{e} \mathscr{D}_{2 k}\right) \phi_{i}^{e}, \phi_{j}^{f}\right\rangle_{\partial \Omega_{l}^{e}}$

\subsection{Elemental notation}

$e(i)$ the elemental index of the element adjacent to edge $i$ of element $e$

$e(i, j)$ the elemental index of the element adjacent to edge $j$ of element $e(i)$, i.e. the element adjacent to edge $i$ of element $e$

$\underline{\hat{u}}^{e(i)} \quad$ the expansion coefficients associated with element $e(i)$

$\hat{\underline{u}}^{e(i, j)} \quad$ the expansion coefficients associated with element $e(i, j)$

$\zeta_{i}^{e} \quad$ unique edge vector used in the LDG flux associated with edge $i$ of element $e$

\section{FORMULATION}

In this section we introduce the discontinuous Galerkin formulation of the elliptic steady diffusion problem with a variable diffusivity tensor. In Section 3.1 we define the strong and auxiliary forms of the diffusion problem. In Section 3.2 we construct the weak form of the auxiliary problem for the global domain. As is typical for a discontinuous Galerkin formulation we then consider the weak construction at an elemental level as discussed in Section 3.2.1. In the elemental formulation we note that a continuous flux at elemental boundaries is required and this type flux is defined in Section 3.2.3 for two different DG methods: the classical Bassi-Rebay and the local discontinuous Galerkin methods. Finally in Section 3.2.4 we discuss the equivalence between the flux formulation adopted in the current work as compared to the flux definition discussed in the widely cited work of Arnold et al. [9].

\subsection{Problem definition}

We consider the following steady diffusion or Poisson problem in a domain $\Omega$ with boundary $\partial \Omega$, which is decomposed into a region of Dirichlet boundary conditions $\partial \Omega_{\mathrm{D}}$ and a region of Neumann boundary conditions $\partial \Omega_{\mathrm{N}}$

$$
\begin{aligned}
-\nabla \cdot(\mathscr{D} \nabla u(\mathbf{x})) & =f(\mathbf{x}) & & \mathbf{x} \in \Omega \\
u(\mathbf{x}) & =g_{\mathrm{D}}(\mathbf{x}) & & \mathbf{x} \in \partial \Omega_{\mathrm{D}} \\
{[\mathscr{D} \nabla u(\mathbf{x})] \cdot \mathbf{n} } & =g_{\mathrm{N}}(\mathbf{x}) & & \mathbf{x} \in \partial \Omega_{\mathrm{N}}
\end{aligned}
$$

where $\partial \Omega_{\mathrm{D}} \cup \partial \Omega_{\mathrm{N}}=\partial \Omega$ and $\partial \Omega_{\mathrm{D}} \cap \partial \Omega_{\mathrm{N}}=\emptyset$. In the above we also consider the diffusivity tensor $\mathscr{D}$ to be a symmetric positive definite matrix which may vary in space, i.e.

$$
\mathscr{D}=\mathscr{D}(\mathbf{x})=\left[\begin{array}{ll}
\mathscr{D}_{11} & \mathscr{D}_{12} \\
\mathscr{D}_{21} & \mathscr{D}_{22}
\end{array}\right]
$$

and $\mathscr{D}_{12}=\mathscr{D}_{21}$. 
3.1.1. Auxiliary formulation. Equation (1) can be written in auxiliary or mixed form as two first-order differential equations by introducing an auxiliary flux variable $\mathbf{q}$, such that

$$
\mathbf{q}=\mathscr{D} \nabla u(\mathbf{x})
$$

Substituting definition (4) into Equation (1) we obtain

$$
\begin{aligned}
-\nabla \cdot \mathbf{q} & =f(\mathbf{x}) \quad \mathbf{x} \in \Omega \\
\mathbf{q} & =\mathscr{D} \nabla u(\mathbf{x}) \quad \mathbf{x} \in \Omega \\
u(\mathbf{x}) & =g_{\mathrm{D}}(\mathbf{x}) \quad \mathbf{x} \in \partial \Omega_{\mathrm{D}} \\
\mathbf{q} \cdot \mathbf{n} & =g_{\mathrm{N}}(\mathbf{x}) \quad \mathbf{x} \in \partial \Omega_{\mathrm{N}}
\end{aligned}
$$

\subsection{Weak form of the auxiliary formulation}

Taking the inner product of Equations (5) and (6) with test functions $v$ and $\mathbf{w}$, respectively, over the solution domain $\Omega$ we obtain

$$
\begin{aligned}
-\int_{\Omega} v \nabla \cdot \mathbf{q} \mathrm{d} \mathbf{x} & =\int_{\Omega} v f(\mathbf{x}) \mathrm{d} \mathbf{x} \\
\int_{\Omega} \mathbf{w} \cdot \mathbf{q} \mathrm{d} \mathbf{x} & =\int_{\Omega} \mathbf{w} \cdot[\mathscr{D} \nabla u(\mathbf{x})] \mathrm{d} \mathbf{x}=\int_{\Omega} \mathscr{D} \mathbf{w} \cdot \nabla u(\mathbf{x}) \mathrm{d} \mathbf{x}
\end{aligned}
$$

where in the last equation we recall that $\mathscr{D}=\mathscr{D}^{\mathrm{T}}$. We assume $\mathscr{T}(\Omega)$ is a two- or threedimensional tessellation of $\Omega$. Let $\Omega^{e} \in \mathscr{T}(\Omega)$ be a non-overlapping element within the tessellation such that if $e_{1} \neq e_{2}$ then $\Omega^{e_{1}} \cap \Omega^{e_{2}}=\emptyset$. Let $\partial \Omega^{e}$ denote the boundary of the element $\Omega^{e}$ and $N_{\mathrm{el}}$ denote the number of elements (or cardinality) of $\mathscr{T}(\Omega)$. For a two-dimensional problem we define the following two spaces:

$$
\begin{aligned}
& V_{h}:=\left\{v \in L^{2}(\Omega):\left.v\right|_{\left.\Omega^{e} \in P\left(\Omega^{e}\right) \forall \Omega^{e} \in \mathscr{T}\right\}}\right. \\
& \boldsymbol{\Sigma}_{h}:=\left\{\tau \in\left[L^{2}(\Omega)\right]^{2}:\left.\tau\right|_{\Omega^{e}} \in \mathbf{\Sigma}\left(\Omega^{e}\right) \forall \Omega^{e} \in \mathscr{T}\right\}
\end{aligned}
$$

where $P\left(\Omega^{e}\right)=\mathscr{T}_{P}\left(\Omega^{e}\right)$ is the linear polynomial space in a triangular region and $P\left(\Omega^{e}\right)=$ $\mathscr{Q}_{P}\left(\Omega^{e}\right)$ is the bilinear polynomial space for a quadrilateral region, defined as

$$
\begin{aligned}
& \mathscr{T}_{P}\left(\Omega^{e}\right)=\left\{x_{1}^{p} x_{2}^{q} ; 0 \leqslant p+q \leqslant P ;\left(x_{1}, x_{2}\right) \in \Omega^{e}\right\} \\
& \mathscr{Q}_{P}\left(\Omega^{e}\right)=\left\{x_{1}^{p} x_{2}^{q} ; 0 \leqslant p, q \leqslant P ;\left(x_{1}, x_{2}\right) \in \Omega^{e}\right\}
\end{aligned}
$$

Similarly $\boldsymbol{\Sigma}\left(\Omega^{e}\right)=\left[\mathscr{T}_{P}\left(\Omega^{e}\right)\right]^{2}$ or $\boldsymbol{\Sigma}\left(\Omega^{e}\right)=\left[\mathscr{Q}_{P}\left(\Omega^{e}\right)\right]^{2}$. For curvilinear regions the expansions are only polynomials when mapped to a straight-sided standard region [22]. 
Let $v^{e} \in V_{h}$ and $\mathbf{w}^{e} \in \boldsymbol{\Sigma}_{h}$ denote scalar and vector test functions, respectively, defined on an element $\Omega^{e}$. The integral forms of Equations (9) and (10) then reduce to finding $u^{e} \in V_{h}$ and $\mathbf{q}^{e} \in \Sigma_{h}$ such that

$$
\begin{aligned}
-\int_{\Omega^{e}} v^{e} \nabla \cdot \mathbf{q}^{e} \mathrm{~d} \mathbf{x} & =\int_{\Omega^{e}} v^{e} f(\mathbf{x}) \mathrm{d} \mathbf{x} \quad \forall v^{e} \in V_{h} \\
\int_{\Omega^{e}} \mathbf{w}^{e} \cdot \mathbf{q}^{e} \mathrm{~d} \mathbf{x} & =\int_{\Omega^{e}} \mathscr{D} \mathbf{w}^{e} \cdot \nabla u^{e}(\mathbf{x}) \mathrm{d} \mathbf{x} \quad \forall \mathbf{w}^{e} \in \mathbf{\Sigma}_{h}
\end{aligned}
$$

We note that the above system is not solvable as every element is now independent of each other. In the standard Galerkin approach the global expansion is chosen to enforce sufficient continuity, which is typically $C^{0}$ for second-order problems, and then a global assembly procedure [22] is necessary to combine the elemental contributions into a global description. However in the discontinuous Galerkin formulation, continuity of flux of the primitive and auxiliary variables is enforced between the elemental boundaries. To illustrate the type of flux continuity adopted in the DG method we continue the problem formulation at an elemental level as given in Equations (11) and (12).

3.2.1. Elemental formulation. The application of the divergence theorem to the individual elemental contributions given by Equations (11) and (12) leads to

$$
\begin{gathered}
\int_{\Omega^{e}} \nabla v^{e} \cdot \mathbf{q}^{e} \mathrm{~d} \mathbf{x}-\int_{\partial \Omega^{e}} v^{e}\left(\mathbf{q}^{e} \cdot \mathbf{n}^{e}\right) \mathrm{d} s=\int_{\Omega^{e}} v^{e} f \mathrm{~d} \mathbf{x} \\
\int_{\Omega^{e}} \mathbf{w}^{e} \cdot \mathbf{q}^{e} \mathrm{~d} \mathbf{x}=-\int_{\Omega^{e}}\left(\nabla \cdot \mathscr{D} \mathbf{w}^{e}\right) u^{e} \mathrm{~d} \mathbf{x}+\int_{\partial \Omega^{e}}\left(\left[\mathscr{D} \mathbf{w}^{e}\right] \cdot \mathbf{n}^{e}\right) u^{e} \mathrm{~d} s
\end{gathered}
$$

In Equations (13) and (14), we note that the values of $u^{e}$ and $\mathbf{q}^{e}$ are required on the boundary of each element. In the absence of a direct enforcement of elemental continuity through the expansion space definition, the local approximation will be discontinuous at the boundary between two elements. We therefore denote a continuous flux on the boundary as $\tilde{u}^{e}$ for the 'flux' of the variable $u^{e}$ and $\tilde{\mathbf{q}}^{e}$ for the 'flux' of the variable $\mathbf{q}^{e}$. The discontinuous Galerkin formulation on every element can now be expressed as

$$
\begin{gathered}
\int_{\Omega^{e}}\left(\nabla v^{e} \cdot \mathbf{q}^{e}\right) \mathrm{d} \mathbf{x}-\int_{\partial \Omega^{e}} v^{e}\left(\mathbf{n}^{e} \cdot \tilde{\mathbf{q}}^{e}\right) \mathrm{d} s=\int_{\Omega^{e}} v^{e} f \mathrm{~d} \mathbf{x} \\
\int_{\Omega^{e}}\left(\mathbf{w}^{e} \cdot \mathbf{q}^{e}\right) \mathrm{d} \mathbf{x}=-\int_{\Omega^{e}}\left(\nabla \cdot \mathscr{D} \mathbf{w}^{e}\right) u^{e} \mathrm{~d} \mathbf{x}+\int_{\partial \Omega^{e}}\left(\left[\mathscr{D} \mathbf{w}^{e}\right] \cdot \mathbf{n}^{e}\right) \tilde{u}^{e} \mathrm{~d} s
\end{gathered}
$$

Alternatively we can apply the divergence theorem going back the other way to obtain an equivalent analytic form

$$
\begin{gathered}
-\int_{\Omega^{e}} v^{e}\left(\nabla \cdot \mathbf{q}^{e}\right) \mathrm{d} \mathbf{x}+\int_{\partial \Omega^{e}} v^{e}\left(\mathbf{n}^{e} \cdot\left[\mathbf{q}^{e}-\tilde{\mathbf{q}}^{e}\right]\right) \mathrm{d} s=\int_{\Omega^{e}} v^{e} f \mathrm{~d} \mathbf{x} \\
\int_{\Omega^{e}}\left(\mathbf{w}^{e} \cdot \mathbf{q}^{e}\right) \mathrm{d} \mathbf{x}=\int_{\Omega^{e}} \mathbf{w}^{e} \cdot \mathscr{D} \nabla u^{e} \mathrm{~d} \mathbf{x}+\int_{\partial \Omega^{e}}\left(\left[\mathscr{D} \mathbf{w}^{e}\right] \cdot \mathbf{n}^{e}\right)\left[\tilde{u}^{e}-u^{e}\right] \mathrm{d} s
\end{gathered}
$$


3.2.2. Stabilization. As we shall explore further in Section 4.1, stabilization is necessary when using the Bassi-Rebay boundary fluxes. It can, however, also be introduced when other fluxes are applied.

Typically stabilization can be introduced as a arbitrary penalization of the jump between the solution along elemental boundaries. To incorporate this into the formulation we introduce the notation $e(l) ; 1 \leqslant l \leqslant N_{b}^{e}$ to denote the elements adjacent to edge $l$. This notation is highlighted in Figure 1 where we show the adjacent element numbers $e(1), e(2)$ and $e(3)$ associated with edges $l=1,2$ and 3 of triangular element $e=e(0)$.

We can now introduce the stabilization factor $\eta \int_{\partial \Omega_{l}^{e}} v^{e}\left(u^{e}-u^{e(l)}\right) \mathrm{d} s$ which penalizes the jump of the primitive function between elemental regions, where $\partial \Omega_{l}^{e}$ is the boundary of edge $l$ of element $e$. This type of stabilization was previously adopted in the LDG formulation presented in Reference [4] and originally used in the SIPG method [2], where $\eta$ was also considered as a function of the edge on which the jump was being penalized.

The weak discontinuous Galerkin elemental formulation (15) and (16) is modified to

$$
\begin{gathered}
\int_{\Omega^{e}}\left(\nabla v^{e} \cdot \mathbf{q}^{e}\right) \mathrm{d} \mathbf{x}-\int_{\partial \Omega^{e}} v^{e}\left(\mathbf{n}^{e} \cdot \tilde{\mathbf{q}}^{e}\right) \mathrm{d} s+\eta \sum_{l=1}^{N_{b}^{e}} \int_{\partial \Omega_{l}^{e}} v^{e}\left(u^{e}-u^{e(l)}\right) \mathrm{d} s=\int_{\Omega^{e}} v^{e} f \mathrm{~d} \mathbf{x} \\
\int_{\Omega^{e}}\left(\mathbf{w}^{e} \cdot \mathbf{q}^{e}\right) \mathrm{d} \mathbf{x}=-\int_{\Omega^{e}}\left(\nabla \cdot \mathscr{D} \mathbf{w}^{e}\right) u^{e} \mathrm{~d} \mathbf{x}+\int_{\partial \Omega^{e}}\left(\left[\mathscr{D} \mathbf{w}^{e}\right] \cdot \mathbf{n}^{e}\right) \tilde{u}^{e} \mathrm{~d} s
\end{gathered}
$$

where if $\eta=0$, Equation (19) reduces to Equation (15). A similar modification can also be applied to Equation (17). Throughout this work, we will consider only this form of stabilization.

We note that in Reference [23] an alternative jump term was presented using the lifting operator $r_{e}$ defined as

$$
\int_{\Omega} r_{e}(\phi) \cdot \tau \mathrm{d} x=-\int_{\partial \Omega_{l}^{e}} \phi \cdot\{\tau\} \mathrm{d} s \quad \forall \tau \in \Sigma_{h}, \quad \phi \in\left[L^{1}(e)\right]^{2}
$$

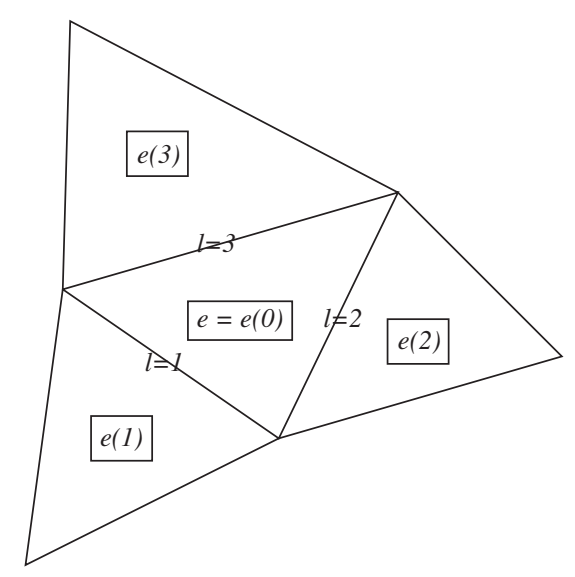

Figure 1. Definition of element numbering $e(l)$ which share an edge $l$ with element $e$. 
where $\Omega$ is the domain over which the tessellation $\mathscr{T}_{h}$ is defined, $\partial \Omega_{l}^{e}$ denotes an edge within that tessellation (which may be owned by one element or may be shared by two adjacent elements) and the curly brackets indicate a jump. The penalization of a variable $\phi$ is given by the expression $-\eta_{e} r_{e}(\phi)$ which uses the aforementioned operator. Once again, a free parameter $\eta_{e}$ allows this stabilizing factor to be tuned for the problem under consideration. As we will demonstrate in the next section, this new stabilizing factor can be incorporated into the flux definition.

3.2.3. Elemental boundary flux definition. Similar to the work of Arnold et al. [9], we define the local discontinuous Galerkin (LDG) flux from which we can automatically obtain the BassiRebay choice. However, in contrast with the form adopted in Reference [9] which couples every components of the auxiliary variables $\mathbf{q}$, we construct an alternative approach where each component of $\mathbf{q}$ remains uncoupled.

We can now define the continuous boundary fluxes $\tilde{u}^{e}$ and $\tilde{\mathbf{q}}^{e}$ as

$$
\left.\tilde{u}^{e}\right|_{\partial \Omega_{i}^{e}}=\left.\left.\alpha_{u}\right|_{i} ^{e} u^{e}\right|_{\partial \Omega_{i}^{e}}+\left.\left.\beta_{u}\right|_{i} ^{e} u^{e(i)}\right|_{\partial \Omega_{i}^{e}}
$$

and

$$
\left.\tilde{\mathbf{q}}^{e}\right|_{\partial \Omega_{i}^{e}}=\left.\left.\alpha_{q}\right|_{i} ^{e} \mathbf{q}^{e}\right|_{\partial \Omega_{i}^{e}}+\left.\left.\beta_{q}\right|_{i} ^{e} \mathbf{q}^{e(i)}\right|_{\partial \Omega_{i}^{e}}
$$

From a consistency point of view, fluxes across an edge shared by $e$ and $e(i)$ should satisfy the constraints $\left.\alpha_{u}\right|_{i} ^{e}+\left.\beta_{u}\right|_{i} ^{e}=1$ and $\left.\alpha_{q}\right|_{i} ^{e}+\left.\beta_{q}\right|_{i} ^{e}=1$ where $\left.\alpha_{u}\right|_{i} ^{e},\left.\quad \beta_{u}\right|_{i} ^{e},\left.\alpha_{q}\right|_{i} ^{e},\left.\beta_{q}\right|_{i} ^{e}$ are realvalued scalars. Under this constraint, we define $\left.\alpha_{u}\right|_{i} ^{e},\left.\quad \beta_{u}\right|_{i} ^{e},\left.\alpha_{q}\right|_{i} ^{e},\left.\quad \beta_{q}\right|_{i} ^{e}$ by first introducing a reference vector $\zeta_{i}^{e}$ along each edge $i$ of element $e$ which is unique along an edge in the sense that $\zeta_{i}^{e}=\zeta_{j}^{f}$ if edge $i$ in element $e$ is adjacent to edge $j$ of element $f$. We can now adopt the following form for the coefficients:

$$
\begin{aligned}
& \left.\alpha_{u}\right|_{i} ^{e}=\frac{1}{2}-\left.\zeta_{i}^{e} \cdot \mathbf{n}^{e}\right|_{\partial \Omega_{i}^{e}} \\
& \left.\beta_{u}\right|_{i} ^{e}=\frac{1}{2}-\left.\zeta_{i}^{e} \cdot \mathbf{n}^{e(i)}\right|_{\partial \Omega_{i}^{e}} \\
& \left.\alpha_{q}\right|_{i} ^{e}=\frac{1}{2}+\left.\zeta_{i}^{e} \cdot \mathbf{n}^{e}\right|_{\partial \Omega_{i}^{e}} \\
& \left.\beta_{q}\right|_{i} ^{e}=\frac{1}{2}+\left.\zeta_{i}^{e} \cdot \mathbf{n}^{e(i)}\right|_{\partial \Omega_{i}^{e}}
\end{aligned}
$$

We observe that the sign of the averaging in $\left.\alpha_{u}\right|_{i} ^{e}$ and $\left.\alpha_{q}\right|_{i} ^{e}$ (as well as $\left.\beta_{u}\right|_{i} ^{e}$ and $\left.\beta_{q}\right|_{i} ^{e}$ ) are reversed to introduce a 'flip-flop' nature of the fluxes where the bias of the continuous flux for $\tilde{u}^{e}$ is reversed to that for the continuous flux of $\tilde{\mathbf{q}}^{e}$. Finally, we also note that the stabilization described in Section 3.2.2 can be incorporated directly into the continuous flux by the following modification to Equations (25) and (26):

$$
\left.\alpha_{q}\right|_{i} ^{e}=\frac{1}{2}+\left.\zeta_{i}^{e} \cdot \mathbf{n}^{e}\right|_{\partial \Omega_{i}^{e}}-\eta \frac{\left.\left.u^{e}\right|_{\partial \Omega_{i}^{e}} n^{e}{ }_{j}\right|_{\partial \Omega_{i}^{e}}}{\left.q_{j}^{e}\right|_{\partial \Omega_{i}^{e}}} \quad j=1,2,3
$$




$$
\left.\beta_{q}\right|_{i} ^{e}=\frac{1}{2}+\left.\zeta_{i}^{e} \cdot \mathbf{n}^{e(i)}\right|_{\partial \Omega_{i}^{e}}-\eta \frac{\left.\left.u^{e(i)}\right|_{\partial \Omega_{i}^{e}} n_{j}^{e(i)}\right|_{\partial \Omega_{i}^{e}}}{\left.q_{j}^{e(i)}\right|_{\partial} \Omega_{i}^{e}} \quad j=1,2,3
$$

The use of Equations (27) and (28) in Equation (15) or (17), is equivalent to applying Equations (25) and (26) in Equation (19).

We can further express $\zeta$ (briefly dropping the subscript and superscript notation) in terms of its magnitude $|\zeta|$ and an angle vector $\mathbf{e}_{\theta}=[\cos \theta \sin \theta]^{\mathrm{T}}$ where $\theta=\tan ^{-1}\left(\zeta_{1} / \zeta_{2}\right)$, i.e. $\zeta=|\zeta| \mathbf{e}_{\theta}$. Adopting this form when $|\zeta|=0$ and $\eta=0$, we regain the classic (unstabilized) Bassi-Rebay scheme. Alternatively if $|\zeta| \neq 0$ and $\eta=0$ we obtain the family of LDG schemes. Setting $\left|\zeta_{i}^{e}\right|=1 / 2$ and

$$
\left.\mathbf{e}_{\theta}\right|_{i} ^{e}= \begin{cases}\left.\mathbf{n}^{e}\right|_{\partial \Omega_{i}^{e}} & \text { if } e<e(i) \\ -\left.\mathbf{n}^{e}\right|_{\partial \Omega_{i}^{e}} & \text { if } e>e(i)\end{cases}
$$

recovers the 'flip-flop' nature of the LDG flux (see Section 3.3.1) as exhibited in the discontinuous Galerkin LDG formulation for one-dimensional problems [12] and as discussed for multi-dimensional problems in Reference [17]. For a given edge there are still two choices of the vector given by Equation (29) and its negative. As we shall demonstrate in Section 4.1 defining $\zeta_{i}^{e}=1 / 2 \mathbf{e}_{\theta}$ as per Equation (29) can lead to a LDG scheme which has a null space larger than one for the solution of the Poisson equation in a periodic region. From the analysis in this section the increase in the dimension of the null space appears to be related to situations where all the local edge vectors $\left.\mathbf{e}_{\theta}\right|_{i} ^{e}$ in a single element point outwards. To avoid this limitation we can determine the direction of $\zeta_{i}^{e}$ by projecting on to an arbitrary global vector g, i.e.

$$
\zeta_{i}^{e}= \begin{cases}\left.\frac{1}{2} \mathbf{e}^{\theta}\right|_{i} ^{e} & \text { if } \mathbf{g} \cdot \zeta_{i}^{e} \geqslant 0 \\ -\left.\frac{1}{2} \mathbf{e}^{\theta}\right|_{i} ^{e} & \text { if } \mathbf{g} \cdot \zeta_{i}^{e}<0\end{cases}
$$

For a curved edge a similar philosophy to the above can be applied but the value of $\boldsymbol{\alpha}_{q}$ and $\boldsymbol{\beta}_{q}$ will now vary along the edge as the value of the normal varies.

3.2.4. Equivalence of fluxes. The continuous auxiliary fluxes $\left.\tilde{\mathbf{q}}\right|^{e}$, given by Equation (22) together with (25) and (26), are not identical to those proposed in the paper of Arnold et al. [9] and used by other researchers. However, equivalence to the flux form presented in the paper of Arnold et al. [9] is observed when both fluxes are projected into the normal elemental direction as required by Equations (15) and (17). Using the previously introduced notation, the auxiliary fluxes of the paper of Arnold et al. [9], denoted here by a subindex $A$, can be written as

$$
\left.\tilde{\mathbf{q}}_{A}^{e}\right|_{\partial \boldsymbol{\Omega}_{i}^{e}}=\frac{1}{2}\left(\left.\mathbf{q}^{e}\right|_{\partial \Omega_{i}^{e}}+\left.\mathbf{q}^{e(i)}\right|_{\partial \Omega_{i}^{e}}\right)+\zeta_{i}^{e}\left(\left.\left.\mathbf{n}^{e}\right|_{\partial \Omega_{i}^{e}} \cdot \mathbf{q}^{e}\right|_{\partial \Omega_{i}^{e}}+\left.\left.\mathbf{n}^{e(i)}\right|_{\partial \Omega_{i}^{e}} \cdot \mathbf{q}^{e(i)}\right|_{\partial \Omega_{i}^{e}}\right)
$$

Inserting Equations (25) and (26) into Equation (22) we can write the auxiliary flux adopted in the current work as

$$
\left.\tilde{\mathbf{q}}^{e}\right|_{\partial \Omega_{i}^{e}}=\frac{1}{2}\left(\left.\mathbf{q}^{e}\right|_{\partial \Omega_{i}^{e}}+\left.\mathbf{q}^{e(i)}\right|_{\partial \Omega_{i}^{e}}\right)+\left.\left(\left.\zeta_{i}^{e} \cdot \mathbf{n}^{e}\right|_{\partial \Omega_{i}^{e}}\right) \mathbf{q}^{e}\right|_{\partial \Omega_{i}^{e}}+\left.\left(\left.\zeta_{i}^{e} \cdot \mathbf{n}^{e(i)}\right|_{\partial \Omega_{i}^{e}}\right) \mathbf{q}^{e(i)}\right|_{\partial \Omega_{i}^{e}}
$$


Recalling that $\left.\mathbf{n}^{e}\right|_{\partial \Omega_{i}^{e}}=-\left.\mathbf{n}^{e(i)}\right|_{\partial \Omega_{i}^{e}}$ the inner product of the normal $\left.\mathbf{n}^{e}\right|_{\partial \Omega_{i}^{e}}$ with Equation (31) as

$$
\begin{aligned}
\left.\left.\mathbf{n}^{e}\right|_{\partial \Omega_{i}^{e}} \cdot \tilde{\mathbf{q}}_{A}^{e}\right|_{\partial \Omega_{i}^{e}}= & \left.\frac{1}{2} \mathbf{n}^{e}\right|_{\partial \Omega_{i}^{e}} \cdot\left(\left.\mathbf{q}^{e}\right|_{\partial \Omega_{i}^{e}}+\left.\mathbf{q}^{e(i)}\right|_{\partial \Omega_{i}^{e}}\right) \\
& +\left(\left.\mathbf{n}^{e}\right|_{\partial \Omega_{i}^{e}} \cdot \zeta_{i}^{e}\right)\left(\left.\left.\mathbf{n}^{e}\right|_{\partial \Omega_{i}^{e}} \cdot \mathbf{q}^{e}\right|_{\partial \Omega_{i}^{e}}\right) \\
& -\left(\left.\mathbf{n}^{e}\right|_{\partial \Omega_{i}^{e}} \cdot \zeta_{i}^{e}\right)\left(\left.\left.\mathbf{n}^{e}\right|_{\partial \Omega_{i}^{e}} \cdot \mathbf{q}^{e}\right|_{\partial \Omega_{i}^{e}}\right)
\end{aligned}
$$

Equivalently the inner product of the normal, $\left.\mathbf{n}^{e}\right|_{\partial \Omega_{i}^{e}}$, with Equation (32) is

$$
\begin{aligned}
\left.\left.\mathbf{n}^{e}\right|_{\partial \Omega_{i}^{e}} \cdot \tilde{\mathbf{q}}_{A}^{e}\right|_{\partial \Omega_{i}^{e}}= & \left.\frac{1}{2} \mathbf{n}^{e}\right|_{\partial \Omega_{i}^{e}} \cdot\left(\left.\mathbf{q}^{e}\right|_{\partial \Omega_{i}^{e}}+\left.\mathbf{q}^{e(i)}\right|_{\partial \Omega_{i}^{e}}\right) \\
& +\left(\left.\zeta_{i}^{e} \cdot \mathbf{n}^{e}\right|_{\partial \Omega_{i}^{e}}\right)\left(\left.\left.\mathbf{n}^{e}\right|_{\partial \Omega_{i}^{e}} \cdot \mathbf{q}^{e}\right|_{\partial \Omega_{i}^{e}}\right) \\
& -\left(\left.\zeta_{i}^{e} \cdot \mathbf{n}^{e}\right|_{\partial \Omega_{i}^{e}}\right)\left(\left.\left.\mathbf{n}^{e}\right|_{\partial \Omega_{i}^{e}} \cdot \mathbf{q}^{e(i)}\right|_{\partial \Omega_{i}^{e}}\right)
\end{aligned}
$$

Clearly the first term corresponds to the classical Bassi-Rebay averaging and is the same in Equations (33) and (34). The individual contributions, $\zeta_{i}^{e}\left(\left.\left.\mathbf{n}^{e}\right|_{\partial \Omega_{i}^{e}} \cdot \mathbf{q}\right|_{\partial \Omega_{i}^{e}}\right)$ and $\left.\left(\left.\zeta_{i}^{e} \cdot \mathbf{n}^{e}\right|_{\partial \Omega_{i}^{e}}\right) \mathbf{q}\right|_{\partial \Omega_{i}^{e}}$, to the average fluxes (31) and (32) are not identical, but their projection into the normal edge direction of these terms are equivalent as illustrated in Figure 2. It is important to stress that the continuous auxiliary flux evaluation $\left.\tilde{\mathbf{q}}^{e}\right|_{\partial \Omega_{i}^{e}}$, using Equation (32) is more easily implemented than the corresponding continuous auxiliary flux evaluation $\left.\tilde{\mathbf{q}}_{A}^{e}\right|_{\partial \Omega_{i}^{e}}$, using Equation (31) since it only involves the inner products $\left(\left.\zeta_{i}^{e} \cdot \mathbf{n}^{e}\right|_{\partial \Omega_{i}^{e}}\right)$ and $\left(\left.\zeta_{i}^{e} \cdot \mathbf{n}^{e(i)}\right|_{\partial \Omega_{i}^{e}}\right)$. The scalar values from these inner products immediately implies that each vector components is decoupled from each other. In contrast, Equation (31) couples the difference components of vector $\left.\mathbf{q}^{e}\right|_{\partial \Omega_{i}^{e}}$ and $\left.\mathbf{q}^{e(i)}\right|_{\partial \Omega_{i}^{e}}$ and so does not permit each component of $\left.\tilde{\mathbf{q}}^{e}\right|_{\partial \Omega_{i}^{e}}$ to be individually evaluated. Although the

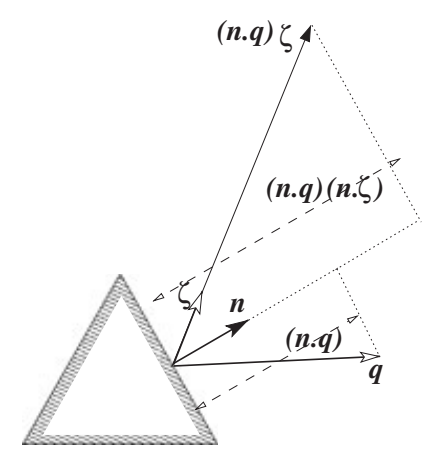

(a)

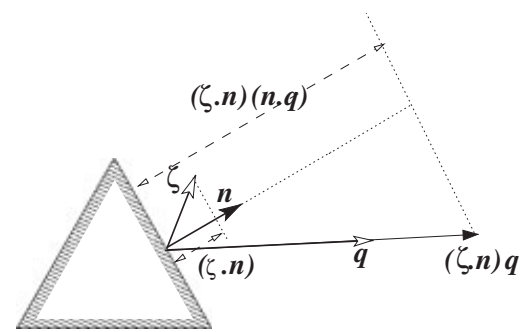

(b)

Figure 2. Graphical equivalence of the projected two-dimensional LDG component of the auxiliary fluxes such that the equality $(\mathbf{n} \cdot \mathbf{q})\left(\mathbf{n} \cdot \zeta_{i}^{e}\right)=\left(\zeta_{i}^{e} \cdot \mathbf{n}\right)(\mathbf{n} \cdot \mathbf{q})$ is numerically preserved in our implementation:

(a) form adopted in Arnold et al. [9]; and (b) form adopted in this work. 
later projection of this flux into the normal direction would allow the components to be treated independently.

3.2.5. Boundary condition enforcement. Up to this point we have only considered the elemental formulation and how to enforce flux continuity between the solution variable and the auxiliary fluxes. It is important to understand how boundary conditions may be enforced, as it modifies some of the properties of the final primal matrix system which is solved.

There are two fundamental mechanisms to enforce Dirichlet boundary conditions. The first is 'strong enforcement' or 'lifting' and the second is 'weak enforcement.' Strong enforcement of the boundary conditions is accomplished through a global lifting of the boundary conditions. We can demonstrate the global lifting process by the following example. Assume we wish to solve the matrix system

$$
\mathbb{A} \underline{x}=\underline{f} \quad \text { where } \underline{x}(\partial \Omega)=\underline{g}(\partial \Omega)
$$

Since this is a linear problem we can decompose $\underline{x}$ into a known solution $\underline{x}^{\mathscr{D}}$ and an unknown homogeneous solution $\underline{x}^{\mathscr{H}}$ such that

$$
\underline{x}=\underline{x}^{\mathscr{D}}+\underline{x}^{\mathscr{U}} \quad \underline{x}^{\mathscr{D}}(\partial \Omega)=\underline{g}(\partial \Omega), \quad \underline{x}^{\mathscr{H}}(\partial \Omega)=0
$$

We can then insert this decomposition into Equation (35) and since $\underline{x}^{\mathscr{D}}$ is a known solution (typically represented in the discrete approximation space) we can put this contribution on the right-hand side to obtain the 'lifted' or 'homogeneous' problem

$$
\mathbb{A}^{\mathscr{H} \mathscr{H}} \underline{x}^{\mathscr{H}}=\underline{f}^{\mathscr{H}}-\left(\mathbb{A}^{\mathscr{H} \mathscr{H}}+\mathbb{A}^{\mathscr{H} \mathscr{D}}\right) \underline{x}^{\mathscr{D}} \quad \text { where } \underline{x}^{\mathscr{D}}(\partial \Omega)=0
$$

where the matrix superscripts $\mathscr{H}$ and $\mathscr{D}$ denote the degrees of freedom associated with the homogeneous (zero on Dirichlet boundaries) and Dirichlet (non-zero on Dirichlet boundaries) degrees of freedom, respectively. In solving the homogeneous problem (36) we therefore only consider discrete expansions which are defined to be zero on a Dirichlet boundary. This reduces the number of global degrees of freedom when compared to the weak enforcement of Dirichlet boundary conditions. As in standard continuous Galerkin finite elements, the value of the approximation on the boundary is 'exact' up to the lifting operator projection error.

Weak enforcement is accomplished through the introduction of the boundary flux term $\tilde{u}^{e}$ in Equation (16) for those elements adjacent to a Dirichlet boundary. This can be implemented either by direct substitution or through the use of 'ghost' elements surrounding the boundary elements in which the solution on the ghost elements is equal to the boundary condition. The potential implementation advantage of 'ghost' elements is that the boundary flux term $\tilde{u}^{e}$ is computed as it would be for any other element/element interface. A few things to note concerning the weak enforcement are that:

(1) Unlike the strong enforcement, weak enforcement does not remove boundary degrees of freedom from the resultant matrix problem which has to be solved.

(2) Weak enforcement (as the name suggests) does not require that the boundary condition be met exactly, but consistently approximated. That is, the boundary condition value is reached in the limit of increasing spatial resolution and the order of convergence is the same as the convergence of the interior scheme. 
(3) One must take care to guarantee that the boundary condition value is applied in an LDG formulation, i.e. that the flip-flop is designed so that the specified boundary values are introduced into the matrix problem.

Arnold et al. [9], as well as most DG practitioners, typically apply a weak enforcement of Dirichlet boundary conditions.

Neumann or natural boundary conditions are handled in an analogous manner to the weak enforcement in general. The Neumann boundary value is substituted directly into elements adjacent to Neumann boundaries as the boundary flux term $\tilde{\mathbf{q}}^{e}$ in Equation (15).

\subsection{Discrete matrix representation}

To get a better appreciation of the implementation of the different DG approaches we now consider the matrix representation of Equations (15)-(16) and (17)-(18) which is more amenable to a numerical implementation of the method. We start by approximating $u^{e}(\mathbf{x})$ and $\mathbf{q}^{e}(\mathbf{x})=$ $\left[q_{1}, q_{2}\right]^{\mathrm{T}}$ by a finite expansion in terms of the basis $\phi_{j}^{e}(\mathbf{x})$ of the form

$$
u^{e}(\mathbf{x})=\sum_{j=1}^{N_{u}^{e}} \phi_{j}^{e}(\mathbf{x}) \underline{\hat{u}}^{e}[j] \quad q_{k}^{e}(\mathbf{x})=\sum_{j=1}^{N_{q}^{e}} \phi_{j}^{e}(\mathbf{x}) \underline{\hat{q}}_{k}^{e}[j]
$$

3.3.1. Matrix form of the auxiliary equations. Following a standard Galerkin formulation we set the scalar test functions $v^{e}$ to be represented by $\phi_{i}^{e}(\mathbf{x})$ where $i=1, \ldots, N_{u}^{e}$, and let our vector test function $\mathbf{w}^{e}$ be represented by $\mathbf{e}_{k} \phi_{i}$ where $\mathbf{e}_{1}=[1,0]^{\mathrm{T}}$ and $\mathbf{e}_{2}=[0,1]^{\mathrm{T}}$. Inserting the finite expansion of the trial functions into Equation (15) with the flux form (25)-(26), the equation for every test function $\phi_{i}$ becomes

$$
\begin{aligned}
\sum_{j=1}^{N_{q}^{e}} & {\left[\left(\frac{\partial \phi_{i}^{e}}{\partial x_{1}}, \phi_{j}^{e}\right)_{\Omega^{e}} \underline{\hat{q}}_{1}^{e}[j]+\left(\frac{\partial \phi_{i}^{e}}{\partial x_{2}}, \phi_{j}^{e}\right)_{\Omega^{e}} \underline{\hat{q}}_{2}^{e}[j]\right] } \\
& -\left.\sum_{l=1}^{N_{b}^{e}} \alpha_{q}\right|_{l} ^{e} \sum_{j=1}^{N_{q}^{e}}\left\langle\phi_{i}^{e},\left[n_{1}^{e} \underline{q}_{1}^{e}[j]+n_{2}^{e} \underline{q}_{2}^{e}[j]\right]\right\rangle_{\partial \Omega_{l}^{e}} \\
- & \left.\sum_{l=1}^{N_{b}^{e}} \beta_{q}\right|_{l} ^{e} \sum_{j=1}^{N_{q}^{e}}\left\langle\phi_{i}^{e},\left[n_{1}^{e} \underline{q}_{1}^{e(l)}[j]+n_{2}^{e} \underline{q}_{2}^{e(l)}[j]\right]\right\rangle_{\partial \Omega_{l}^{e}}=\left(\phi_{i}^{e}, f\right)_{\Omega^{e}}
\end{aligned}
$$

Here we recall that $e(l)$ denotes the adjacent elements which share a common edge with element $e$ as illustrated in Figure 1. Introducing the matrices

$$
\begin{gathered}
\mathbb{D}_{k}^{e}[i, j]=\left(\phi_{i}^{e}, \frac{\partial \phi_{j}^{e}}{\partial x_{k}}\right)_{\Omega^{e}} \\
\mathbb{E}_{k l}^{e, f}[i, j]=\left\langle\phi_{i}^{e}, \phi_{j}^{f} n_{k}^{e}\right\rangle_{\partial \Omega_{l}^{e}}
\end{gathered}
$$


and defining $\underline{f}^{e}[i]=\left(\phi_{i}, f\right)_{\partial \Omega^{e}}$ we can write Equation (37) in matrix form as

$$
\left[\left(\mathbb{D}_{1}^{e}\right)^{\mathrm{T}}\left(\mathbb{D}_{2}^{e}\right)^{\mathrm{T}}\right]\left[\begin{array}{l}
\hat{q}_{1}^{e} \\
\hat{q}_{2}^{e}
\end{array}\right]-\left.\sum_{l=1}^{N_{b}^{e}} \alpha_{q}\right|_{l} ^{e}\left[\mathbb{E}_{1 l}^{e, e} \mathbb{E}_{2 l}^{e, e}\right]\left[\begin{array}{l}
\hat{q}_{1}^{e} \\
\hat{q}_{2}^{e}
\end{array}\right]-\left.\sum_{l=1}^{N_{b}^{e}} \beta_{q}\right|_{l} ^{e}\left[\mathbb{E}_{1 l}^{e, e(l)} \mathbb{E}_{2 l}^{e, e(l)}\right]\left[\begin{array}{l}
\hat{q}_{1}^{e(l)} \\
\hat{q}_{2}^{e(l)}
\end{array}\right]=\underline{f}^{e}
$$

In the matrix system (38) the matrix $\mathbb{D}^{e}$ denotes the elemental weak derivative commonly used in standard Galerkin implementations. On the other hand, the matrix $\mathbb{E}_{k l}^{e, f}$ is a type of mass matrix evaluated on an element edge and projected in the normal component direction $n_{k}$. We also note that when $e \neq f$ this elemental mass matrix involves the inner product of two, potentially completely different, edge expansions $\phi_{i}^{e}\left(\partial \Omega_{l}^{e}\right)$ and $\phi_{i}^{f}\left(\partial \Omega_{l}^{e}\right)$ (note $\partial \Omega_{l}^{e}=\partial \Omega_{m}^{f}$ when edge $l$ of element $e$ is adjacent to edge $m$ of element $f$ ).

The two DG formulations we are considering differ in their support in the computational domain. Figure 3 shows the role of $\alpha_{q}$ and $\beta_{q}$ in Equation (38) when Bassi-Rebay and a normalized direction LDG fluxes are employed. In Figure 3(a) we present the contribution to the element denoted by a circle when using Bassi-Rebay fluxes. In this case the boundary flux evaluation uses information from both sides of an edge and so the shaded elements are involved. In Figure 3(b) we illustrate the influence on the element denoted by a circle of adopting the normalized direction LDG fluxes. In this case the orientation of the normals (inward or outward facing) is based upon projection against a globally defined orientation vector $\mathbf{g}$. The single element diagram on the right of this figure provides the LDG values of $\beta_{u}$ and $\beta_{q}$ on each edge when Equations (29) and (30) are employed. Note that $\alpha_{u}$ and $\alpha_{q}$ are immediately deducible due to the convex combination requirements. Once again the shaded elements surrounding the element under consideration denote the elements from which non-zero contributions are obtained in Equation (38) (due to the particular $\alpha_{q}$ and $\beta_{q}$ values). We note that not all adjacent elements are involved in the LDG evaluation as dictated by its 'flip-flop' nature.

If we now consider Equation (16) for the $k$ th component of the auxiliary flux, $q_{k}$, we observe that, on insertion of the finite trial basis expansion, we have for every test function $\phi_{i}$

$$
\begin{aligned}
\sum_{j=1}^{N_{q}^{e}}\left(\phi_{i}^{e}, \phi_{j}^{e}\right)_{\Omega^{e}} \underline{\hat{q}}_{k}^{e}[j]= & -\sum_{j=1}^{N_{u}^{e}}\left[\left(\frac{\partial}{\partial x_{1}}\left[\mathscr{D}_{1 k} \phi_{i}^{e}\right]+\frac{\partial}{\partial x_{2}}\left[\mathscr{D}_{2 k} \phi_{i}^{e}\right], \phi_{j}^{e}\right)_{\Omega^{e}}\right] \underline{\hat{u}}^{e}[j] \\
& +\left.\sum_{l=1}^{N_{b}^{e}} \alpha_{u}\right|_{l} ^{e} \sum_{j=1}^{N_{u}^{e}}\left[\left\langle\left(n_{1}^{e} \mathscr{D}_{1 k}+n_{2}^{e} \mathscr{D}_{2 k}\right) \phi_{i}^{e}, \phi_{j}^{e}\right\rangle_{\partial \Omega_{l}^{e}}\right] \underline{\hat{u}}^{e}[j] \\
& +\left.\sum_{l=1}^{N_{b}^{e}} \beta_{u}\right|_{l} ^{e} \sum_{j=1}^{N_{u}^{e}}\left[\left\langle\left(n_{1}^{e} \mathscr{D}_{1 k}+n_{2}^{e} \mathscr{D}_{2 k}\right) \phi_{i}^{e}, \phi_{j}^{e(l)}\right\rangle_{\partial \Omega_{l}^{e}}\right] \underline{\hat{u}}^{e(l)}[j]
\end{aligned}
$$

and introducing the matrices

$$
\begin{aligned}
& \mathbb{M}^{e}[i, j]=\left(\phi_{i}^{e}, \phi_{j}^{e}\right)_{\Omega^{e}} \\
& \tilde{\mathbb{D}}_{k}^{e}[i, j]=\left(\phi_{i}^{e}, \frac{\partial}{\partial x_{1}}\left[\mathscr{D}_{1 k} \phi_{j}^{e}\right]+\frac{\partial}{\partial x_{2}}\left[\mathscr{D}_{2 k} \phi_{j}^{e}\right]\right)_{\Omega^{e}}
\end{aligned}
$$




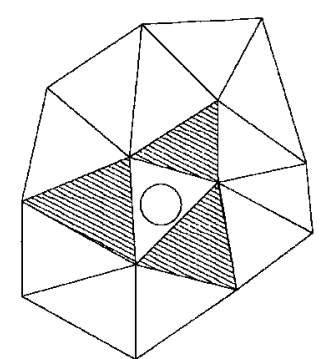

(a)

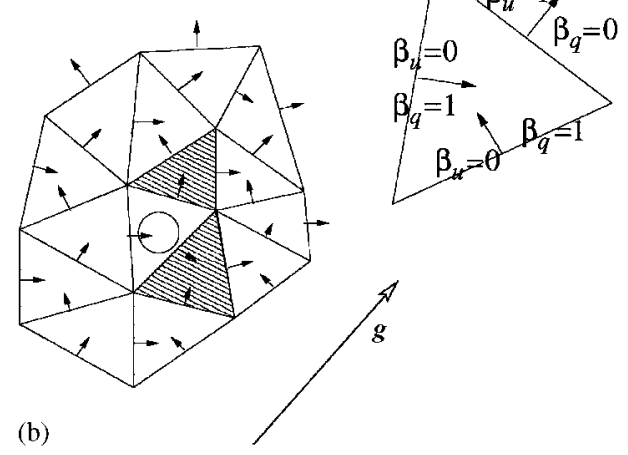

Figure 3. Schematic illustrating the role of $\alpha_{q}=1-\beta_{q}$ and $\beta_{q}$ in Equation (38) when: (a) Bassi-Rebay; and (b) normalized direction LDG fluxes are employed. A complete explanation of the diagram is provided in the text.

$$
\mathbb{F}_{k l}^{e, f}[i, j]=\left\langle\left(n_{1}^{e} \mathscr{D}_{1 k}+n_{2}^{e} \mathscr{D}_{2 k}\right) \phi_{i}^{e}, \phi_{j}^{f}\right\rangle_{\partial \Omega_{l}^{e}}
$$

we can write Equation (39) as

$$
\mathbb{M}^{e} \underline{\hat{q}}_{k}^{e}=-\left(\tilde{\mathbb{D}}_{k}^{e}\right)^{\mathrm{T}} \underline{\hat{u}}^{e}+\left.\sum_{l=1}^{N_{b}^{e}} \alpha_{u}\right|_{l} ^{e} \mathbb{F}_{k l}^{e, e} \underline{\hat{u}}^{e}+\left.\sum_{l=1}^{N_{b}^{e}} \beta_{u}\right|_{l} ^{e} \mathbb{F}_{k l}^{e, e(l)} \underline{\hat{u}}^{e(l)}
$$

In Equation (40), $\mathbb{M}^{e}$ is the element mass matrix used in standard Galerkin formulations. Matrix $\tilde{\mathbb{D}}_{k}^{e}$ denotes the inner product of the divergence of diffusivity tensor by the vector expansion basis for the $k$ th component of the auxiliary flux. We note that, if the diffusivity tensor has the simplified form $\mathscr{D}=v \mathbf{I}$ where $v$ is a constant, then $\tilde{\mathbb{D}}_{k}^{e}=v \mathbb{D}_{k}^{e}$. Finally the matrix $\mathbb{F}_{k l}^{e, f}$ is another edge matrix denoting a type of elemental mass matrix weighted with the $k$ th component of the diffusivity tensor and edge normals. Once again if $\mathscr{D}=v \mathbf{I}$ then $\mathbb{F}_{k l}^{e, f}=v \mathbb{E}_{k l}^{e, f}$. We note that the matrix operation $\left(\mathbb{M}^{e}\right)^{-1} \mathbb{F}_{k l}^{e, e}$ represents the discrete elemental lifting operation (see Section 3.2.5) which 'lifts' or extends the information from edge $l$ of the solution $u^{e}$ into the interior of the element through the action of the inverse mass matrix.

Similar to the example of Figure 3 it is interesting to note the elemental coupling of Equation (40). Therefore in Figure 4 we present a schematic illustrating the role of $\alpha_{u}$ and $\beta_{u}$ in Equation (40) when both Bassi-Rebay and normalized direction LDG fluxes are employed. As indicated by the shaded triangles in Figure 4(a), the region of influence of the Bassi-Rebay fluxes on an element of interest (denoted by the circle) is identical to the stencil shown in Figure 3. For the normalized direction LDG flux we recall the direction of $\zeta_{i}^{e}$ is indicated by the edge arrows in Figure 4(b) and is a consequence of applying Equation (30) against a globally defined orientation vector $\mathbf{g}$. The LDG values of $\alpha_{u}$ and $\beta_{u}$ in this example are such that only one shaded element contributes to the element of interest denoted by the circle in Equation (40). This is the only element which was not used in the LDG flux of Figure 3(b). 


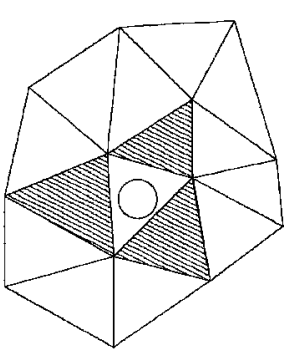

(a)

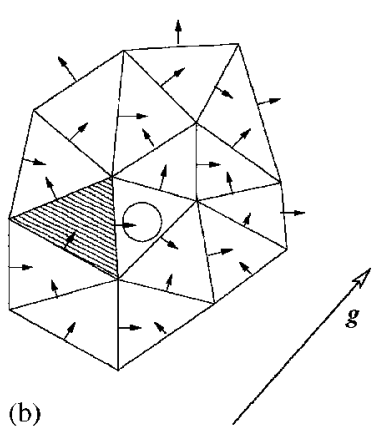

(b)

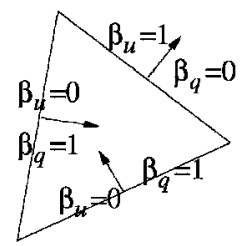

Figure 4. Schematic demonstrating the role of $\alpha_{u}=1-\beta_{u}$ and $\beta_{u}$ in Equation (40) when LDG based upon Equation (29) is employed. A complete explanation of the diagram is provided in the text.

Finally we can also define the elemental matrix representing the inner product with the $k$ th component of the gradient of the expansion basis multiplied by the diffusivity tensor, i.e.

$$
\hat{\mathbb{D}}_{k}^{e}[i, j]=\left(\phi_{i}^{e}, \mathscr{D}_{k 1} \frac{\partial \phi_{j}^{e}}{\partial x_{1}}+\mathscr{D}_{k 2} \frac{\partial \phi_{j}^{e}}{\partial x_{2}}\right)_{\Omega^{e}}
$$

In the absence of any integration errors we note that the adjoint relationship between $\hat{\mathbb{D}}_{k}^{e}$ and $\tilde{\mathbb{D}}_{k}^{e}$ can be expressed as

$$
\hat{\mathbb{D}}_{k}^{e}=-\left(\tilde{\mathbb{D}}_{k}^{e}\right)^{\mathrm{T}}+\sum_{l=1}^{N_{b}^{e}} \mathbb{F}_{k l}^{e, e}
$$

Therefore inserting Equation (41) into Equation (40) we obtain

$$
\mathbb{M}^{e} \hat{q}_{k}^{e}=\hat{\mathbb{D}}_{k}^{e} \underline{\hat{u}}^{e}+\sum_{l=1}^{N_{b}^{e}}\left(\left.\alpha_{u}\right|_{l} ^{e}-1\right) \mathbb{F}_{k l}^{e, e} \underline{\hat{u}}^{e}+\left.\sum_{l=1}^{N_{b}^{e}} \beta_{u}\right|_{l} ^{e} \mathbb{F}_{k l}^{e, e(l)} \underline{\hat{u}}^{e(l)}
$$

which is the discrete matrix representation of Equation (18).

It is useful to note, at this point in our derivation, that the mass matrix $\mathbb{M}^{e}$ in the auxiliary flux equations given by Equation (42) is decoupled at an elemental level. Hence an elemental inversion of the mass matrix allows us to write an explicit equation for the auxiliary flux variable. This will be used in the next section for the derivation of a matrix form of the primal equation. We also recall that the multiplication by the inverse mass matrix acts as a local lifting operator-lifting the influence of the boundary flux terms across the entire elemental expansion.

3.3.2. Matrix form of the primal equation. Building upon the matrix representations of the Section 3.3.1 we can obtain the matrix form of the primal Equation (1). To proceed we insert Equation (42) into Equation (38) to obtain the two-dimensional primal form that corresponds to 
the continuous elemental auxiliary Equations (15) and (18). This matrix system can be written as

$$
\mathbb{K}^{1} \underline{\hat{u}}^{e}+\left.\sum_{m=1}^{N_{b}^{e}} \beta_{u}\right|_{m} ^{e} \mathbb{K}_{m}^{2} \underline{\hat{u}}^{e(m)}-\left.\sum_{l=1}^{N_{b}^{e}} \beta_{q}\right|_{l} ^{e} \mathbb{K}_{l}^{3} \underline{\hat{u}}^{e(l)}-\sum_{l=1}^{N_{b}^{e}} \sum_{m=1}^{N_{b}^{e(l)}} \mathbb{K}_{l m}^{4} \underline{\hat{u}}^{e(l, m)}=\underline{f}^{e}
$$

where we have used the definitions described in the following. $\mathbb{K}^{1}$ denotes the contribution of the elemental region $e$ and is given by

$$
\begin{aligned}
\mathbb{K}^{1}= & {\left[\left(\left(\mathbb{D}_{1}^{e}\right)^{\mathrm{T}}-\left.\sum_{l=1}^{N_{b}^{e}} \alpha_{q}\right|_{l} ^{e} \mathbb{E}_{1 l}^{e, e}\right)\left(\mathbb{M}^{e}\right)^{-1}\left(\hat{\mathbb{D}}_{1}^{e}+\sum_{l=1}^{N_{b}^{e}}\left(\left.\alpha_{u}\right|_{l} ^{e}-1\right) \mathbb{F}_{1 l}^{e, e}\right)\right.} \\
& \left.+\left(\left(\mathbb{D}_{2}^{e}\right)^{\mathrm{T}}-\left.\sum_{l=1}^{N_{b}^{e}} \alpha_{q}\right|_{l} ^{e} \mathbb{E}_{2 l}^{e, e}\right)\left(\mathbb{M}^{e}\right)^{-1}\left(\hat{\mathbb{D}}_{2}^{e}+\sum_{l=1}^{N_{b}^{e}}\left(\left.\alpha_{u}\right|_{l} ^{e}-1\right) \mathbb{F}_{2 l}^{e}\right)\right]
\end{aligned}
$$

The terms $\sum_{i=1}^{2}\left(\mathbb{D}_{i}^{e}\right)^{\mathrm{T}}\left(\mathbb{M}^{e}\right)^{-1} \hat{\mathbb{D}}_{i}^{e}$ correspond to the elemental contribution which typically arises in a standard Galerkin formulation and is recovered when $\mathbb{E}_{k l}^{e, f}=\mathbb{F}_{k l}^{e, f}=0$.

$\mathbb{K} \mathbb{K}_{m}^{2}$ denotes the contributions from the elements immediately adjacent to the edges of element $e$ and is given by

$$
\begin{aligned}
\mathbb{K}_{m}^{2}= & \left.\sum_{m=1}^{N_{b}^{e}} \beta_{u}\right|_{m} ^{e}\left[\left(\left(\mathbb{D}_{1}^{e}\right)^{\mathrm{T}}-\left.\sum_{l=1}^{N_{b}^{e}} \alpha_{q}\right|_{l} ^{e} \mathbb{E}_{1 l}^{e, e}\right)\left(\mathbb{M}^{e}\right)^{-1} \mathbb{F}_{1 m}^{e, e(m)}\right. \\
& \left.+\left(\left(\mathbb{D}_{2}^{e}\right)^{\mathrm{T}}-\left.\sum_{l=1}^{N_{b}^{e}} \alpha_{q}\right|_{l} ^{e} \mathbb{E}_{2 l}^{e, e}\right)\left(\mathbb{M}^{e}\right)^{-1} \mathbb{F}_{2 m}^{e, e(m)}\right]
\end{aligned}
$$

This matrix arises from the contribution of the third term in Equation (42) being inserted into the first two terms of Equation (38).

$\mathbb{K}_{l}^{3}$ denotes the contributions from the elements immediately adjacent to the edges of element $e$ and is given by

$$
\begin{aligned}
\mathbb{K}_{l}^{3}= & \left.\sum_{l=1}^{N_{b}^{e}} \beta_{q}\right|_{l} ^{e}\left[\mathbb{E}_{1 l}^{e, e(l)}\left(\mathbb{M}^{e(l)}\right)^{-1}\left(\hat{\mathbb{D}}_{1}^{e(l)}+\sum_{m=1}^{N_{b}^{e(l)}}\left(\left.\alpha_{u}\right|_{m} ^{e(l)}-1\right) \mathbb{F}_{1 m}^{e(l), e(l)}\right)\right. \\
& \left.+\mathbb{E}_{2 l}^{e, e(l)}\left(\mathbb{M}^{e(l)}\right)^{-1}\left(\hat{\mathbb{D}}_{2}^{e(l)}+\sum_{m=1}^{N_{b}^{e(l)}}\left(\left.\alpha_{u}\right|_{m} ^{e(l)}-1\right) \mathbb{F}_{2 m}^{e(l), e(l)}\right)\right]
\end{aligned}
$$

This matrix arises due to the first two terms of Equation (42) being inserted into the third term of Equation (38). Finally $\mathbb{K}_{l m}^{4}$ denotes the contributions to the primal form of elements adjacent to the edges of the elements adjacent to element $e$ and arises when the third term of 
Equation (42) is inserted into the third term of Equation (38), it is defined as

$$
\mathbb{K}_{l m}^{4}=\left.\sum_{l=1}^{N_{b}^{e}} \beta_{q}\right|_{l} ^{e}\left[\left.\sum_{m=1}^{N_{b}^{e(l)}} \beta_{u}\right|_{m} ^{e(l)}\left[\mathbb{E}_{1 l}^{e, e(l)}\left(\mathbb{M}^{e(l)}\right)^{-1} \mathbb{F}_{1 m}^{e(l), e(l, m)}+\mathbb{E}_{2 l}^{e, e(l)}\left(\mathbb{M}^{e(l)}\right)^{-1} \mathbb{F}_{2 m}^{e(l), e(l, m)}\right]\right]
$$

In this matrix definition we have extended the use of the previously defined superscript notation $e(i)$ to the form $e(i, j)$. This extension $e(i, j)$ is to be understood as the element index of the neighbouring element adjacent to edge $j$ of $e(i)$ which we recall is the element adjacent to edge $i$ of element $e$. Therefore, this involves the elements in two 'halos' surrounding element $e$.

In a continuous Galerkin formulation the global continuity between elemental regions enforces that the information from element $e$ is coupled to the elements adjacent to its immediate edges, although the $C^{0}$ continuous vertex modes typically couple further information from all neighbouring elements. We therefore note that the matrices $\mathbb{K}_{m}^{2}, \mathbb{K}_{l}^{3}$ and $\mathbb{K}_{l m}^{4}$ represent the non-local contributions to the primal form.

Having constructed the matrix form (43) of the primal equation we can now observe that to generate a local discontinuous Galerkin method which has a 'local' influence on adjacent elements we require that either $\left.\beta_{q}\right|_{i} ^{e}$ or $\left.\beta_{u}\right|_{m} ^{e}\left(m=1, \ldots, N_{b}^{e}\right)$ must be identically zero to make $\mathbb{K} \mathbb{l}_{l m}^{4}$ zero (or equivalently either $\left.\alpha_{q}\right|_{i} ^{e}$ or $\left.\alpha_{u}\right|_{m} ^{e}\left(m=1, \ldots, N_{b}^{e}\right)$ have a value of 1$)$. Recalling Equations (24) and (26) we deduce that a necessary condition for the LDG formulation to maintain a local structure is that

$$
\left.\zeta_{i}^{e} \cdot \mathbf{n}^{e}\right|_{\partial \Omega_{i}^{e}}= \pm \frac{1}{2}
$$

The most obvious choice for $\zeta_{i}^{e}$ along edge $\partial \Omega_{i}^{e}$ was previously given in Equation (29) and is

$$
\zeta_{i}^{e}= \pm\left.\frac{1}{2} \mathbf{n}^{e}\right|_{\partial \Omega_{i}^{e}}
$$

Clearly an arbitrary component of the tangent can be added to $\zeta_{i}^{e}$. It would appear that this relatively specific choice of $\zeta_{i}^{e}$ to achieve a local scheme has not been widely discussed. For example Arnold et al. [9] suggest any vector $\zeta$ is suitable. However Cockburn et al. [17] have previously suggested a vector similar to the one defined above. As we shall demonstrate in Section 4.1 the choice of sign of $\zeta_{i}^{e}$ should be normalized using a projection to a global direction similar to Equation (30) to avoid generating undesirable increases in the dimension of the null spaces of the operator.

We note that the definition of the LDG vector using Equations (29) and (30) does not absolutely guarantee a local scheme (where only elements adjacent to ' $e$ ' are used). This arises due to the role of $\left.\beta_{u}\right|_{i} ^{e}$ in the inner summation of the list two lines of Equation (43) where a $\left.\beta_{u}\right|_{i} ^{e} \neq 0$ can arise on any non-local edge and be coupled through $\left.\beta_{q}\right|_{i} ^{e}$ to element $e$. To illustrate this point we build upon the examples of Figures 3 and 4 . In Figure 5 we schematically present the stencil or region of influence with respect to Equation (43) for the classic Bassi-Rebay (left) and the LDG, based upon Equations (29) and (30) (right), schemes. As with previous illustration examples, the triangle at the top of the diagram is to remind the reader of the effect of the normalized direction LDG. Since the classic Bassi-Rebay employs a factor of $1 / 2$ for all $\alpha$ and $\beta$ values, several observations and deductions can be made. First, the classic Bassi-Rebay stencil is quite large with a total footprint of 10 elements. Secondly, the LDG stencil based upon Equations (29) and (30) has a far more compact stencil than the 


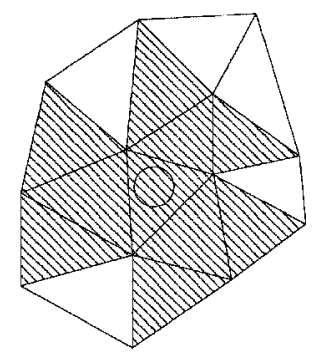

(a)

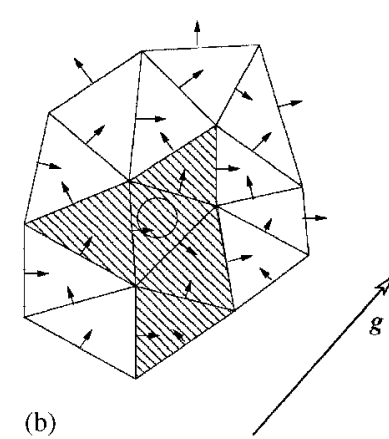

(b)

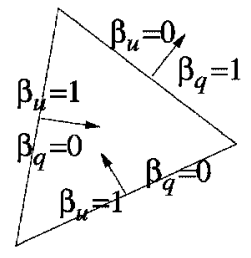

Figure 5. Schematic demonstrating the stencil (region of influence) with respect to Equation (43) classic Bassi-Rebay (left) and the normalized direction LDG based upon Equations (29) and (30) (right). The triangle at the top of the diagram is to remind the reader of the consequences of the LDG choice. The classic Bassi-Rebay scheme employs one-half for all $\alpha$ and $\beta$ values. A complete explanation of the diagram is provided in the text.

Bassi-Rebay stencil. This is due to the 'flip-flop' nature of the convex combination. Thirdly, the LDG stencil is not guaranteed to use information only from neighbouring elements in contrast, for instance, to the formulation by Baumann-Oden [24].

The choice as to whether to set $\beta_{u}$ or $\beta_{q}$ to zero along a solution boundary is important when considering Neumann boundary conditions. In this case we require that $\beta_{q}=1$ otherwise the Neumann boundary flux will not be incorporated into the weak problem. The analogous issue for implementation of Dirichlet boundary conditions depends on whether this condition is enforced through either a penalty or a lifting approach.

\subsection{Polynomial expansion basis}

In the following numerical implementation we have applied a spectral/hp element type discretization which is described in detail in Reference [22]. In this section we describe the orthogonal and $C^{0}$ continuous quadrilateral and triangular expansions within the standard regions which we have adopted.

For a standard quadrilateral region $-1 \leqslant x_{1}, x_{2} \leqslant 1$ a $P$ th order orthogonal polynomial expansion can be defined as the tensor product of Legendre polynomials $L_{p}(x)$ such that

$$
\phi_{i(p q)}\left(x_{1}, x_{2}\right)=L_{p}\left(x_{1}\right) L_{q}\left(x_{2}\right) \quad 0 \leqslant p, q \leqslant P
$$

where the pair $i(p q)$ represents the unique indexing of the $1 \mathrm{D}$ indices $p, q$ to the consecutive list $i$. Analogously the most commonly used hierarchical $C^{0}$ polynomial expansion [22] is based on the tensor product of the integral of Legendre polynomials (or equivalently generalized Jacobi polynomials $\left.P_{p}^{1,1}(x)\right)$ such that

$$
\phi_{i(p q)}\left(x_{1}, x_{2}\right)=\psi_{p}\left(x_{1}\right) \psi_{q}\left(x_{2}\right) \quad 0 \leqslant p, q \leqslant P
$$



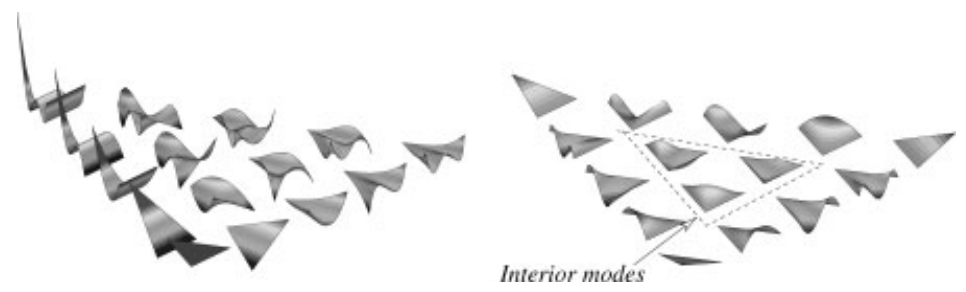

Figure 6. Triangular expansion modes for a $P=4$ order expansion using an orthogonal expansion (left) and a $C^{0}$ continuous expansion (right). The modes in the $C^{0}$ expansion can be identified as either interior (being zero on all boundaries) or boundary modes.

where

$$
\psi_{p}(x)= \begin{cases}\frac{1-x}{2}, & p=0 \\ \frac{1-x}{2} \frac{1+x}{2} P_{p}^{1,1}(x), & 0<p<P \\ \frac{1+x}{2}, & p=P\end{cases}
$$

Within a triangular domain we can use an orthogonal expansion described by, amongst others, Priorol [25], Koornwinder [26] and Dubiner [27]. This expansion is illustrated in Figure 6 (left) and is explicitly defined for $P$ th order expansions in $-1 \leqslant x_{1}, x_{2} ; x_{1}+x_{2} \leqslant 0$ as

$$
\phi_{i(p q)}\left(x_{1}, x_{2}\right)=P_{p}^{0,0}\left(x_{1}\right)\left(\frac{1-x_{2}}{2}\right)^{p} P_{q}^{2 p+1,0}\left(x_{2}\right) \quad 0 \leqslant p, q ; p+q \leqslant P
$$

where $P_{i}^{\alpha, \beta}$ denotes the $i$ th Jacobi polynomial based upon $\alpha$ and $\beta$. A $C^{0}$ extension of this expansion was proposed by Dubiner [27] and is also detailed in Reference [22]. The $C^{0}$ expansion for an expansion of order $P=4$ is shown in Figure 6 (right).

As we will demonstrate in Section 3.5, there are different numerical considerations associated with the choice of orthogonal or $C^{0}$ continuous expansions. The orthogonal expansion, by definition, has a diagonal mass matrix in elemental regions with linear mappings to the standard region. This property can be numerically useful when evaluating the auxiliary variable q. Alternatively, the use of a $C^{0}$ expansion allows the boundary conditions to be efficiently enforced through a global lifting type operation where a known function with exact boundary conditions is lifted out of the problem as discussed in Section 3.2.5. Further, the natural decomposition of the $C^{0}$ basis into interior modes that are zero on the elemental boundaries and boundary modes allows us to use a static condensation technique where the structure of the global matrix system arising from the DG formulation can be used effectively to solve the system as discussed in the next section.

\subsection{Static condensation}

When the global matrix system has a simple block structure, such as a series of decoupled submatrix systems, the static condensation technique is an algebraic manipulation which utilizes 
this structure to simplify the solution of the system. Static condensation or sub-structuring is a technique commonly used in continuous Galerkin methods, particularly for the $p$-type expansions where the construction of many 'interior' or 'bubble' expansions naturally lend themselves to this type of decomposition. If we consider a symmetric matrix problem such as

$$
\left[\begin{array}{ll}
\mathbb{A} & \mathbb{B} \\
\mathbb{B}^{\mathrm{T}} & \mathbb{C}
\end{array}\right]\left[\begin{array}{l}
\underline{u}_{1} \\
\underline{u}_{2}
\end{array}\right]=\left[\begin{array}{l}
\underline{f}_{1} \\
\underline{f}_{2}
\end{array}\right]
$$

the problem can be restated as initially solving for $\underline{u}_{1}$ by considering the sub-matrix problem

$$
\mathbb{S} \underline{u}_{1}=\left[\mathbb{A}-\mathbb{B C}^{-1} \mathbb{B}^{\mathrm{T}}\right] \underline{u}_{1}=\underline{f}_{1}-\mathbb{B} \mathbb{C}^{-1} \underline{f}_{2}
$$

where $\mathbb{S}$ is referred to as the Schur complement. The vector $\underline{u}_{2}$ can then be solved via the sub-matrix problem

$$
\underline{C}_{2}=\underline{f}_{2}-\mathbb{B}^{\mathrm{T}} \underline{u}_{1}
$$

We observe that solving the statically condensed problem is not more efficient than considering the full problem unless $\mathbb{C}^{-1}$ is easy to evaluate. In the case of a $C^{0}$ continuous $p$-type element expansion, the interior or bubble modes have a block diagonal structure in the global matrix and so this matrix has a numerically efficient inverse when compared to the full inverse of the global matrix of equal rank. This point is highlighted in Figure 7 where we schematically illustrate the structure of an elliptic continuous Galerkin matrix. The matrix can be considered as being constructed from block diagonal elemental components which have been ordered into sub-matrices containing just the boundary and interior components. In constructing the global matrix system, a direct assembly procedure involving the matrix $\mathbb{A}$ [22] can be applied which enforces the continuity between the elemental regions on the boundary degrees of freedom. However since interior or bubble modes are, by definition, zero on elemental boundaries they can be considered individually as global degrees of freedom and so the globally assembled matrix maintains the block diagonal structure of the interior-interior sub-block. This matrix can therefore be inverted at the elemental level thereby dramatically reducing the size of the global matrix problem for high-order polynomial expansions. It is also possible to apply a similar philosophy to a cluster of elements where the interior degrees of freedom are defined to be modes which are zero on the boundary of the elemental cluster.

If we are to consider the direct inversion of the discontinuous problem then application of the static condensation technique may also be desirable. However, in the discontinuous Galerkin formulation there is direct enforcement of the continuity of the elemental expansions across elements. We therefore might consider adopting an expansion which has an diagonal elemental mass matrix such as the tensor product of Legendre polynomials. We note that the global matrix in the DG scheme is the same rank as the sum of the elemental degrees of freedom and so we no longer have a global assembly procedure denoted by $\mathbb{A}$. However in the DG scheme we introduce elemental boundary fluxes which couple adjacent elements and lead to off-diagonal components in the matrix structure.

At this point it is not evident whether we can still apply the static condensation technique to the discontinuous Galerkin formulation. Indeed, it is not until we adopt a $C^{0}$ continuous expansion, typically used in the continuous Galerkin formulation, that we recover an appropriate structure to apply this technique. To appreciate why it is possible to use static condensation 

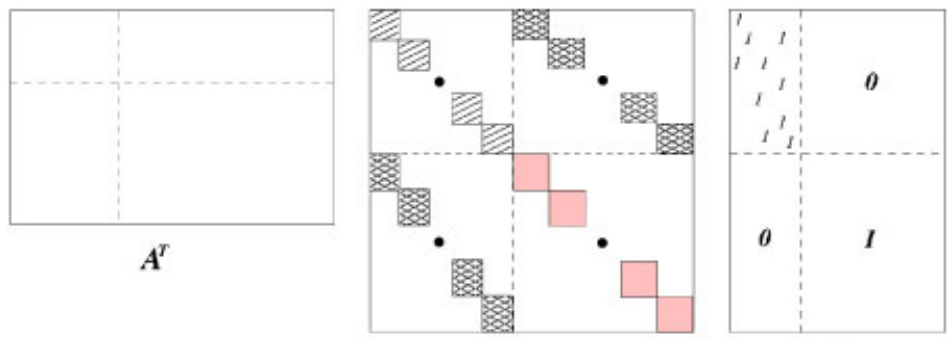

$\boldsymbol{A}$

Figure 7. Schematic construction of continuous matrix system. In the continuous Galerkin system, the matrix can be interpreted as block diagonal systems which are globally assembled through pre- and post-multiplying by a restriction matrix $\mathbb{A}$.

we observe that the definition of $\mathbb{E}_{k l}^{e, f}$ and $\mathbb{E}_{k l}^{e, f}$ are purely dependent upon the support of the expansion along the elemental boundaries. Since by definition all interior modes are zero along the elemental boundaries, $\mathbb{E}_{k l}^{e, f}$ and $\mathbb{F}_{k l}^{e, f}$ are necessarily zero for all interior modes.

\section{MATRIX ANALYSIS}

In Section 4.1 we investigate the null space of the primal matrix Equation (43) and the conditioning of this matrix in Section 4.2.

\subsection{Null space of the Laplacian operator}

4.1.1. Bassi-Rebay flux. It is well known that the Bassi-Rebay choice of boundary flux $\alpha_{u}=\beta_{u}=\alpha_{q}=\beta_{q}=\frac{1}{2}$ with no stabilization leads to a Laplace operator with spurious modes due to an enriched null space [9]. In this section we solve the Poisson problem using a uniform mesh of triangular and quadrilateral elements in a periodic domain. The null space is evaluated using double precision with the general matrix eigenvalue routine in the LAPACK library applied to Equation (43). An eigenvalue was defined to be in the null space if the magnitude of the eigenvalue was less than $1 \times 10^{-13}$. The eigenvector associated with the zero eigenvalue provides a set of expansion coefficients and so the corresponding eigenfunction was determined by evaluating the expansion at a series of quadrature points in the solution domain.

Figure 8 shows representative null space functions which arise at different polynomial orders when considering a periodic region $\left[0 \leqslant x_{1}, x_{2} \leqslant 1\right]$ subdivided into eight equally shaped triangles. In this figure we plot the function and its derivatives with respect to $x_{1}$ and $x_{2}$ which are used to evaluate the auxiliary fluxes. Table I shows the size of the numerical evaluated null space for this mesh as a function of polynomial orders and we note that for the triangular expansion the dimension of the null space increases with polynomial order.

We recall from Equations (21) and (22) that the primitive and auxiliary fluxes for the BassiRebay fluxes are given by the average of the values of the function or the relevant derivative either side of an elemental interface. From an inspection of Figure 8 we observe that the average fluxes will be zero at any point on the elemental boundary and so these non-zero modes in the null space have no global coupling from $\tilde{u}$ between elements. A similar property 

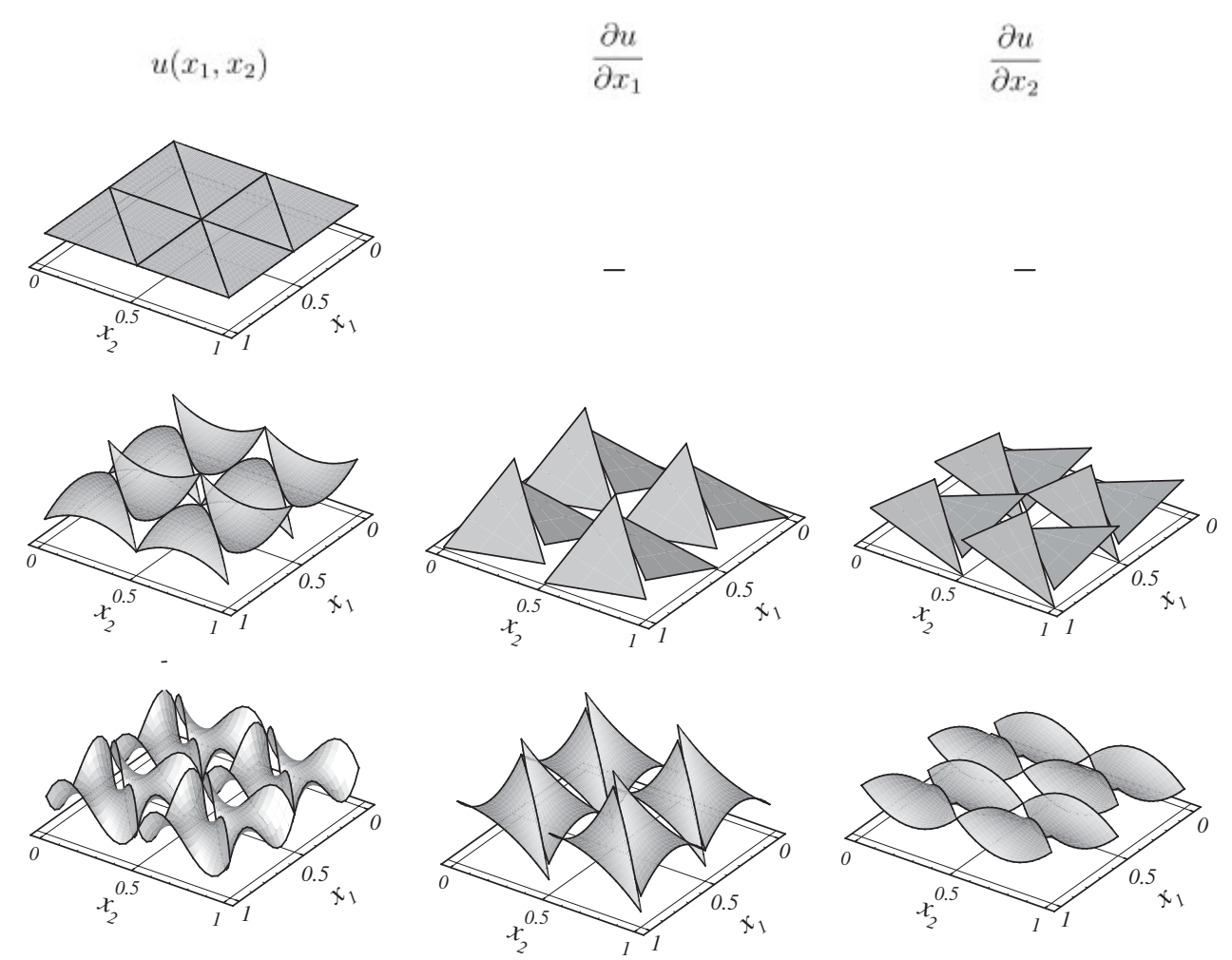

Figure 8. Representative null space eigenmodes and their derivatives (arbitrarily scaled) for $P=1$ (top), $P=2$ (middle), and $P=3$ (bottom) polynomial order triangular expansions.

Table I. Numerical evaluation of the dimension of the null space $\left(\left|\lambda_{i}\right| \leqslant\right.$ $1 \times 10^{-13}$ ) using Bassi-Rebay fluxes for different polynomial order expansions $P$ in the domain shown in Figures 8 and 9 using similar shaped triangular and quadrilateral elements.

\begin{tabular}{lllllrrrr}
\hline Poly Order. $P$ & 1 & 3 & 5 & 7 & 9 & 11 & 13 & 15 \\
Dim. of tri. null space & 1 & 3 & 3 & 3 & 5 & 5 & 5 & 7 \\
Dim. of Quad. null space & 4 & 4 & 4 & 4 & 4 & 4 & 4 & 4 \\
Poly Order. $P$ & 2 & 4 & 6 & 8 & 10 & 12 & 14 & 16 \\
Dim. of tri. null space & 2 & 2 & 4 & 4 & 4 & 6 & 6 & 6 \\
Dim. of quad. null space & 4 & 4 & 4 & 4 & 4 & 4 & 4 & 4 \\
\hline
\end{tabular}

appears to hold for the derivatives of the null space modes which decouples the contribution of the continuous flux of the auxiliary variable $\tilde{\mathbf{q}}$.

Figure 9 shows some representative null space functions for a quadrilateral discretization of the domain $0 \leqslant x_{1}, x_{2} \leqslant 1$ into four quadrilateral elements. This null space also contains the constant mode which is not shown. In contrast to Figure 8 we observe that, whilst the value 

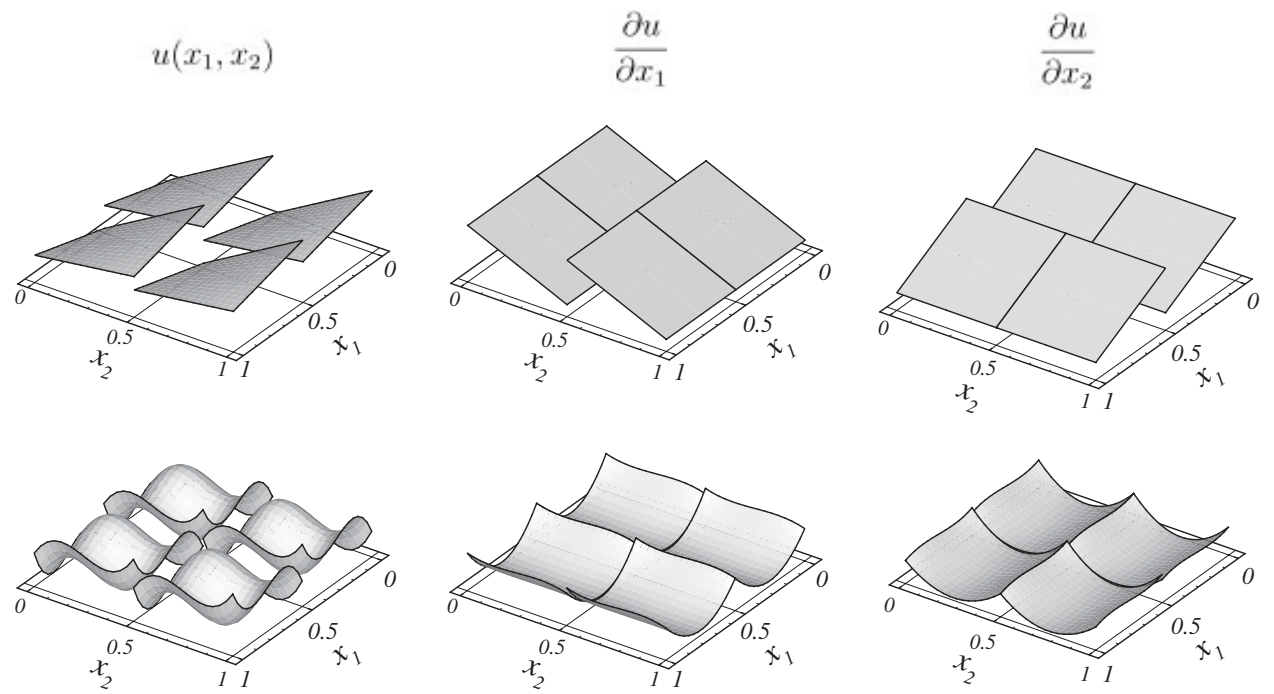

Figure 9. Representative null space eigenmodes (arbitrarily scaled) for $P=1$ (top) and $P=3$ (bottom) polynomial order quadrilateral expansions.

of the primitive functions have the property that the values are equal and opposite along the elemental boundaries (up to the constant mode), the derivatives of the function in the null space no longer have this property. Therefore the average auxiliary flux, $\tilde{\mathbf{q}}^{e}$ along the elemental boundaries is not always identically zero. However the integral

$$
\int_{\partial \Omega^{e}} \tilde{\mathbf{q}}^{e} \cdot \mathbf{n}^{e} \mathrm{~d} s
$$

is zero since the value of the auxiliary average fluxes is equal along boundaries where the elemental normal is equal and opposite. Finally we note from Table I that the dimension of the null space for quadrilateral discretizations considered does not increase with polynomial order.

4.1.2. $L D G$ flux. To complement our investigation of the null space of the Bassi-Rebay flux we also consider the null space of the LDG flux with no stabilization. In Section 3.3 we argued that the only choice of the edge vector $\left.\zeta\right|_{i} ^{e}$ that leads to a 'local' discontinuous Galerkin formulation, which has similar coupling as the standard Galerkin method, is to define $\left.\zeta\right|_{i} ^{e}$ as in Equation (44). However, in the following test we observe that the direction of the unique vector along a given edge is important since a choice where all vectors are either internal or external to a local element leads to an undesirable increase in the dimension of the null space.

To illustrate this point we consider the computational domains used in the null space studies in the previous section. We then prescribe the vector $\left.\zeta\right|_{i} ^{e}$ using only Equation (29). This means that the element with the lowest global identity has $\left.\zeta\right|_{i} ^{e}$ vectors which are all aligned with the inwards normal direction of this element and so $\beta_{u}=0$. Similarly the element with largest global identity has $\left.\zeta\right|_{i} ^{e}$ vectors which are aligned with the outwards normal to the element and so $\beta_{q}=0$. As with the Bassi-Rebay fluxes and shown in Table II, this definition of $\left.\zeta\right|_{i} ^{e}$ leads to a null space which increases in dimension with polynomial order for the triangular mesh and 
Table II. Numerical evaluation of the dimension of the null space $\left(\lambda_{i} \leqslant\right.$ $1 \times 10^{-13}$ ) using non-normalized LDG fluxes for different polynomial order expansions $P$ in the domain shown in Figures 8 and 9 using similar shaped triangular and quadrilateral elements.

\begin{tabular}{llllrrrrr}
\hline Poly Order. $P$ & 1 & 3 & 5 & 7 & 9 & 11 & 13 & 15 \\
Dim. of tri. null space & 3 & 5 & 7 & 9 & 11 & 13 & 15 & 17 \\
Dim. of Quad. null space & 4 & 4 & 4 & 4 & 4 & 4 & 4 & 4 \\
Poly Order. $P$ & 2 & 4 & 6 & 8 & 10 & 12 & 14 & 16 \\
Dim. of tri. null space & 4 & 6 & 8 & 10 & 12 & 14 & 16 & 18 \\
Dim. of quad. null space & 4 & 4 & 4 & 4 & 4 & 4 & 4 & 4 \\
\hline
\end{tabular}
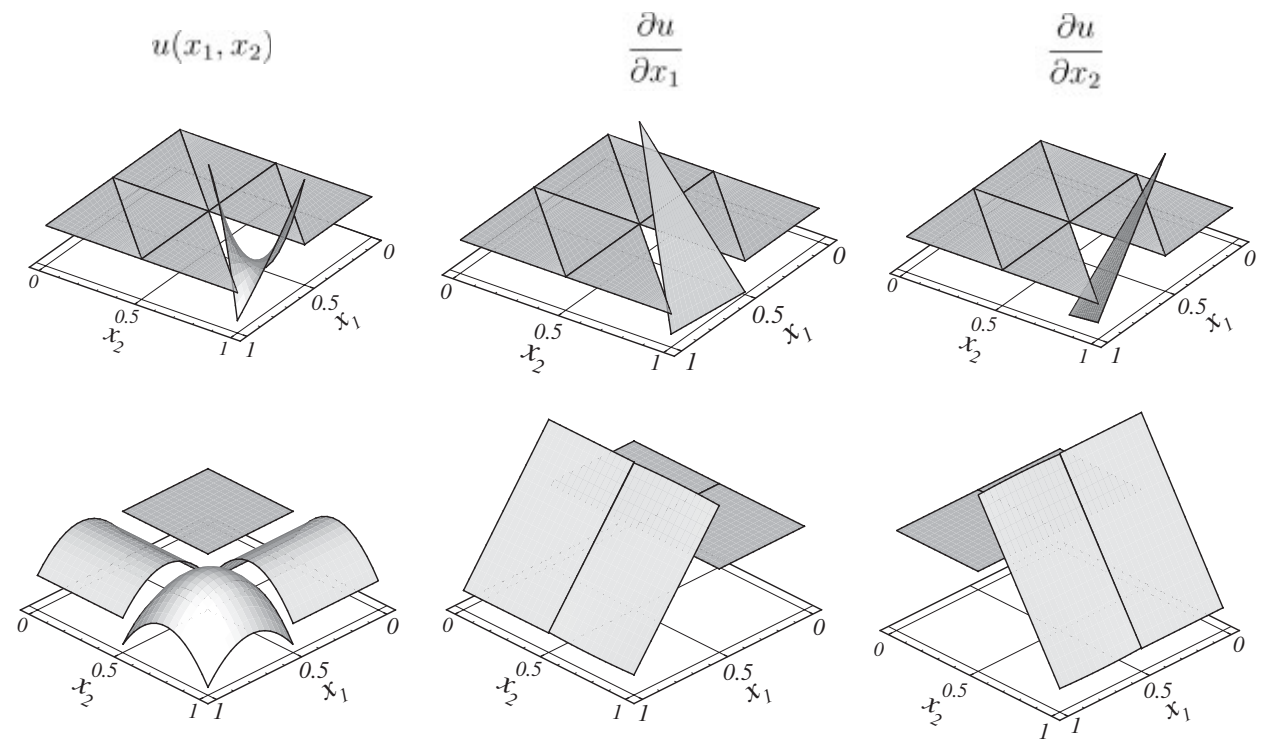

Figure 10. Representative null space eigenmodes (arbitrarily scaled) for polynomial order $P=2$ in a triangular elements (top) in a quadrilateral elements (bottom).

gives a fixed null space of dimension 4 for the quadrilateral mesh considered. A representative mode of the null space and the elemental derivatives when $P=2$ are also shown in Figure 10. We note that the null space is non-zero in the element with highest global number.

\subsection{Condition number scaling}

To complete the numerical investigation of the continuous and discontinuous Galerkin formulations we consider the conditioning of both the matrices of the system and their Schur complement. In all the following computations we have again considered the Laplacian operator in the region $0 \leqslant x_{1}, x_{2} \leqslant 1$ with periodic boundary conditions. We numerically determined the $L_{2}$ condition number as the ratio of the maximum to minimum eigenvalues. We have excluded the zero eigenvalue corresponding to the constant solution. 
As we have observed in Section 4.1, if stabilization is not applied to the DG method with Bassi-Rebay fluxes, spurious modes exist due to the presence of a non-physical null space of the discrete system. To suppress these spurious modes we can apply stabilization as discussed in Section 3.2.2. We therefore start by considering the role of the stabilization factor $\eta$ in Equations (27) and (28) on the condition number of the system. In Figure 11 we show plots of the $L_{2}$ condition number of the full system as a function of the stabilization factor $\eta$ for both the Bassi-Rebay flux and the LDG flux where the direction normalization of Equation (30) has been applied. In this test we divided the computational domain into four equispaced quadrilateral elements. Similar trends were observed for a triangular discretization where each quadrilateral region was subdivided into two triangular elements. Castillo [14] theoretically and numerically analysed a range of DG method for different fluxes as a function of the stabilization factor normalized by the mesh space $h$. Although this work did not consider Bassi-Rebay flux, it was noted that the condition number should asymptotically vary linearly with condition number for larger stabilization factors in the relatively similar interior penalty (IP) approach. This property is observed for both fluxes considered in Figure 11. For the IP method he also found that the condition number was inversely proportional to the stabilization factor for small values of $\eta$. This property would also appear to be present in the Bassi-Rebay flux. Certainly we would expect an increase in the condition number as the stabilization factor tends to zero and more spurious modes of the Bassi-Rebay fluxes are introduced into the system.

However, in contrast to the findings of Castillo [14], we observe that for the normalized direction LDG method the condition number is constant as the stabilization factor tends to zero. If the direction is not normalized we observed in Section 4.1.2 that spurious modes can enter the system and therefore we could expect an increase of the condition number for small stabilization factors.

This observation appears to be consistent with observations made in $[17,18]$ where stabilization is required in the weak enforcement of boundary conditions. As we are examining a periodic domain and using the direction normalized LDG as presented, we have eliminated the source of the conditioning problem, and hence see that the condition number does not grow as the penalization is taken to zero. We have observed a similar behaviour when using Dirichlet boundary conditions directly enforced through a global lifting of a known function satisfying the boundary conditions.

In our next set of tests we consider the scaling of the $L_{2}$ condition number as a function of the characteristic $h$ of the elemental regions, the aspect ratio of elemental regions and the polynomial order applied within every element. We start by considering a series of hierarchical meshes as shown in Figure 12. The computational domain is now sub-divided into 4, 16, 64 and 256 equal square elements as shown in Figures 12(a)-(d). To analyse the effect of the aspect ratio of different meshes we have also used a series of meshes of quadrilateral elements which are refined into the bottom left-hand corner as shown in Figures 12(e)-(h). The smallest elements of these meshes correspond to the smallest elements of the uniformly discretized cases. The meshes shown in Figures 12(e)-(h) have elements with maximum aspect ratios of $1,2,4$ and 8, respectively.

Figures 13(a) and (b) show the $L_{2}$ condition number of both the Bassi-Rebay flux and the normalized direction LDG flux for polynomial orders in the range $1 \leqslant P \leqslant 5$. In these plots we have taken the size of the elements along each edge in Figures 12(a)-(d) as a measure of the element spacing. Over the $h$-range considered we observe that the Bassi-Rebay flux scales as $O\left(h^{2}\right)$ for linear polynomial orders but this rate is somewhat slower at higher polynomial 

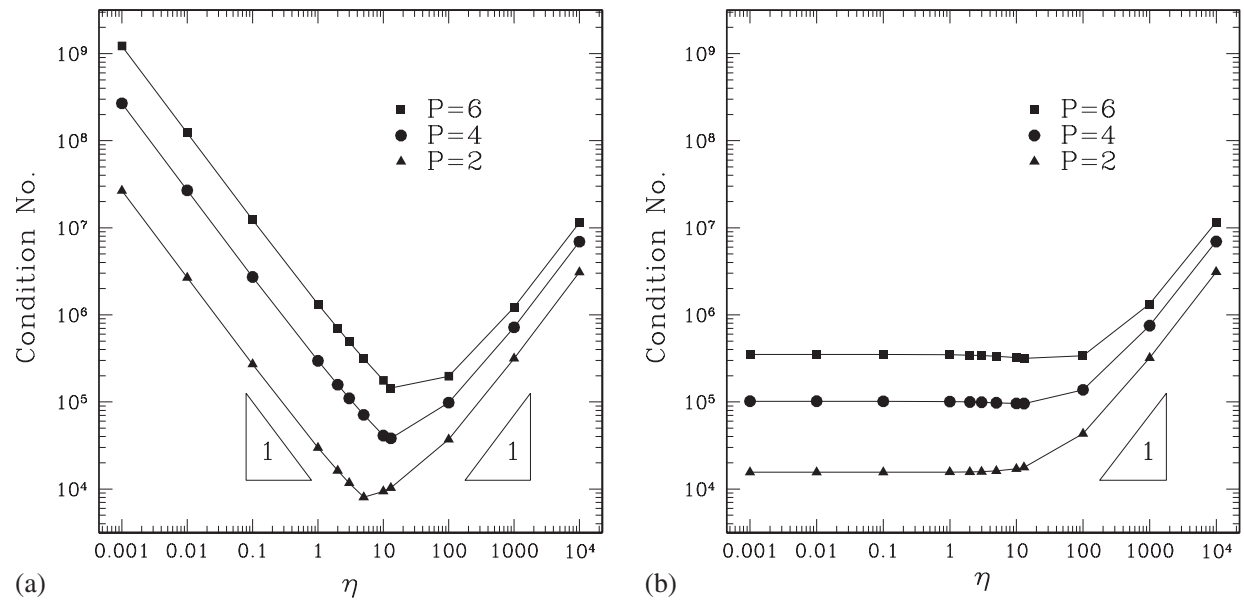

Figure 11. $L_{2}$ condition number scaling for the stabilization factor $\eta$ for polynomial orders of $P=2,4$ and 6 for the: (a) Bassi-Rebay flux and (b) the normalized direction LDG flux. The domain consists of four equal $r$ quadrilateral elements in $0 \leqslant x_{1}, x_{2} \leqslant 1$.

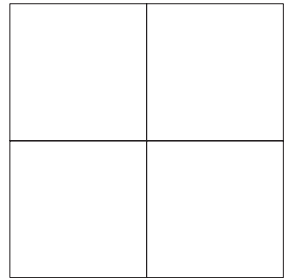

(a)

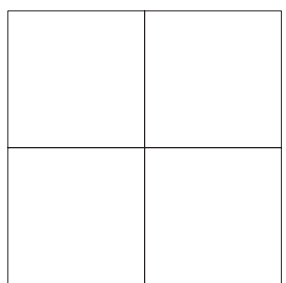

(e)

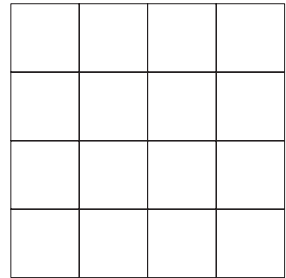

(b)

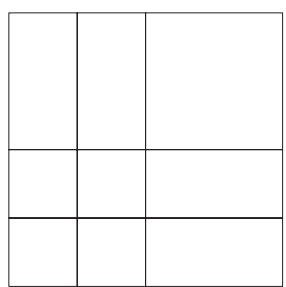

(f)

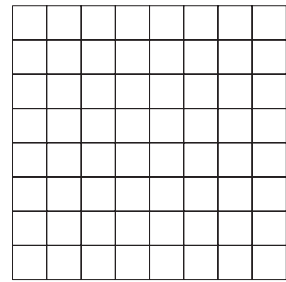

(c)

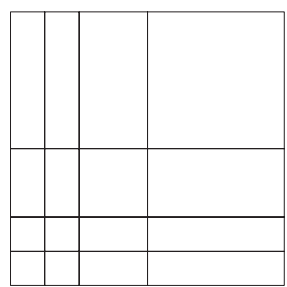

(g)

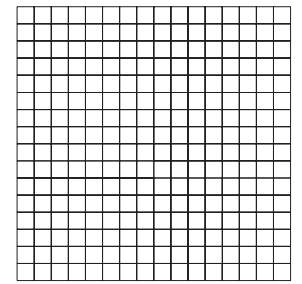

(d)

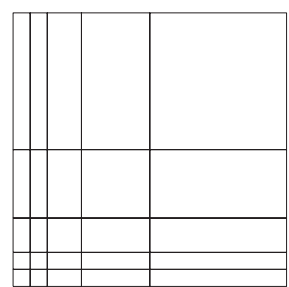

(h)

Figure 12. Computational domains in $0 \leqslant x_{1}, x_{2} \leqslant 1$ used for condition number tests. AR denotes the maximum aspect ratio of the elements: (a) $2 \times 2$; (b) $4 \times 4$; (c) $4 \times 4$; (d) $16 \times 16$; (e) $\mathrm{AR}=1$; (f) $\mathrm{AR}=2$; (g) $\mathrm{AR}=4$; and (h) $\mathrm{AR}=8$.

orders. Somewhat more surprisingly we also observe that on these meshes the LDG flux at a higher than linear polynomial order does not vary with $h$. For the form of expansion basis presented in this work, a slower than logarithmic scaling with $h$ has previously been observed in the conditioning of the Schur complement system of the continuous Galerkin system [22]. 

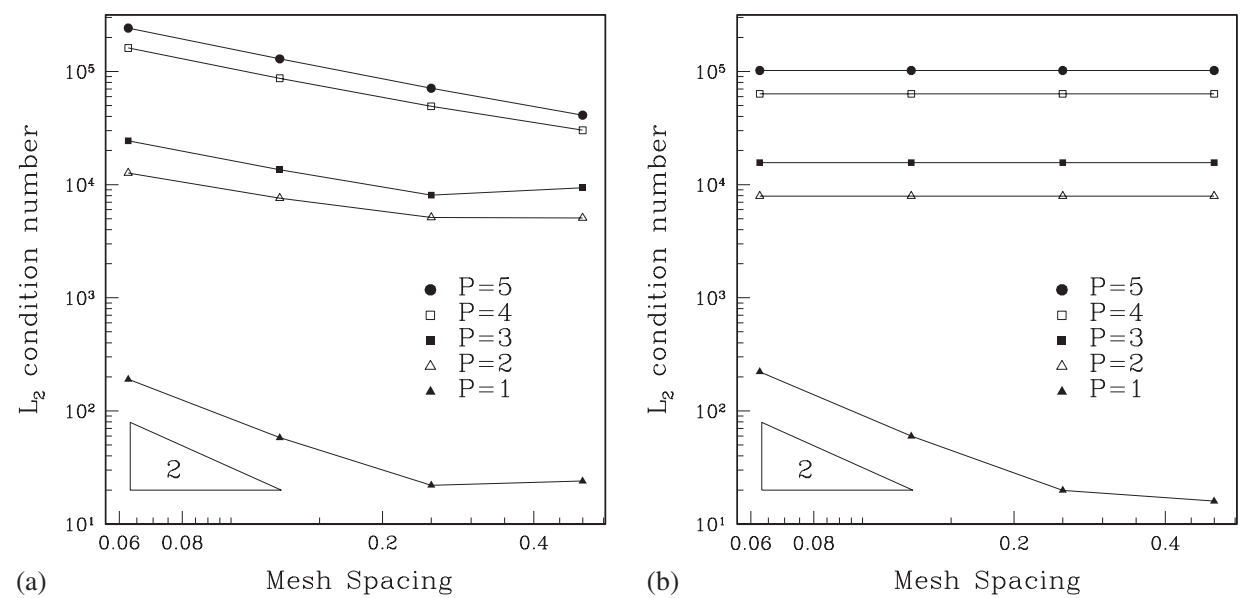

Figure 13. $L_{2}$ condition number scaling as a function of mesh spacing $h$ for the full system using: (a) Bassi-Rebay flux with $\eta=10$; (b) the normalised direction LDG flux. The computational domain adopted are shown in Figures 12(a)-(d).
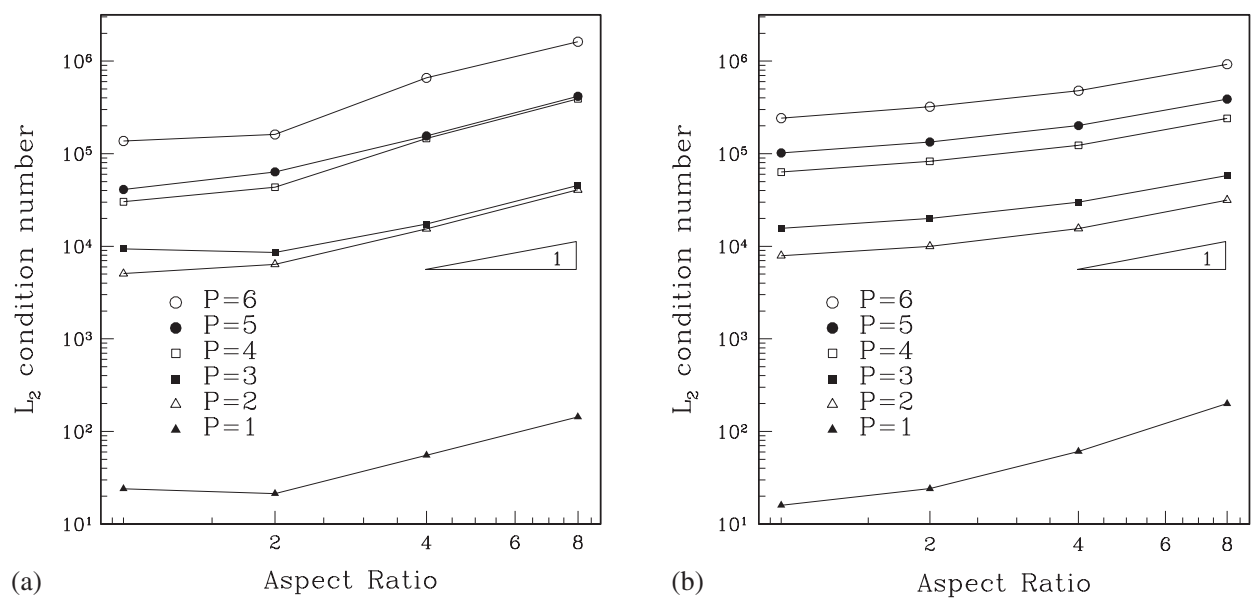

Figure 14. $L_{2}$ condition number scaling as a function of combined mesh spacing $h$ and aspect ratio AR for the full system using: (a) Bassi-Rebay flux with $\eta=10$; and (b) the normalized direction LDG flux. The domain adopted are shown in Figures 12(e)-(h).

Figures 14(a) and (b) show a similar test to that shown in Figure 13 but on the non-uniform meshes of Figures $12(\mathrm{e})-(\mathrm{h})$ and for $1 \leqslant P \leqslant 6$. In this test we plot the growth of the condition number as a function of the maximum aspect ratio (AR) of the mesh. We note however that the smallest element size in the mesh is necessarily also modified as the aspect ratio is changed. At higher polynomial orders the Bassi-Rebay flux demonstrates a linear growth with aspect ratio. Similarly the LDG flux also demonstrates a linear growth rate with aspect ratio. This is in contrast with the $h$-scaling tests. 

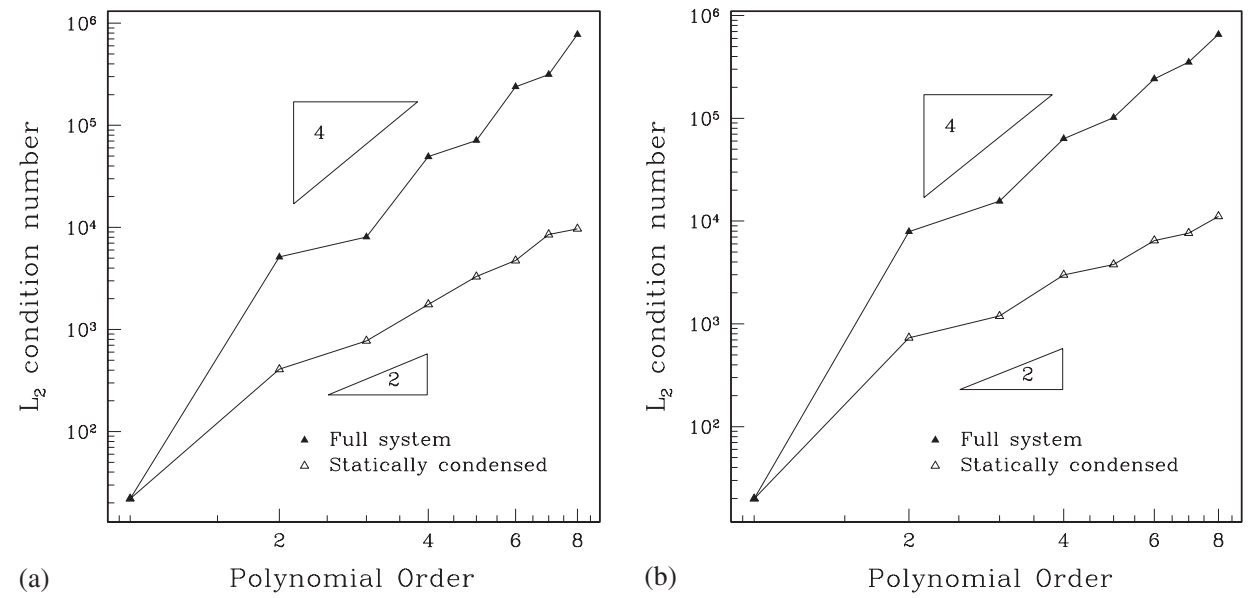

Figure 15. $L_{2}$ condition number scaling as a function of polynomial order for the full system and Schur complements using: (a) Bassi-Rebay flux with $\eta=10$; and (b) the normalized direction LDG flux. The computational domain consisted of sixteen equal square elements in $0 \leqslant x_{1}, x_{2} \leqslant 1$.

In our final test we calculate the scaling of the $L_{2}$ condition number as a function of polynomial order on the $4 \times 4$ mesh of uniform quadrilateral regions shown in Figure 12(b). The results of this test for both the full matrix system and the Schur complement system obtained from static condensation is shown in Figure 15. Similar to the continuous Galerkin formulation [22], we observe an $O\left(P^{4}\right)$ scaling with polynomial order, $P$, of the $L_{2}$ condition number for the full system as opposed to an $O\left(P^{2}\right)$ scaling for the Schur complement system.

\section{EXAMPLES}

To conclude our investigation we consider two elliptic problems. The solutions of the first has smooth derivatives but that of the second has not.

In our first test case, shown in Figure 16, we consider an unstructured triangular discretization around the British Isles. Within this computational domain we solve the Helmholtz problem $\nabla^{2} u-\lambda u=f$, with $\lambda=1$, exact Dirichlet boundary conditions and an exact solution

$$
u\left(x_{1}, x_{2}\right)=\sin \left(\frac{1}{4 \pi \sqrt{\left(x_{1}-a\right)^{2}+\left(x_{2}-b\right)^{2}}}\right)
$$

where the constants $a$ and $b$ have been chosen to centre the solution on London. Figure 16(b) shows the $H_{1}$ error of the numerical discretization as a function of polynomial order using a standard continuous Galerkin (CG) formulation and a DG formulation using Bassi-Rebay (BR) and LDG fluxes. A stabilization factor of $\eta=10$ was used in the Bassi-Rebay formulation. Static condensation was applied in the solution technique for all cases. On the semi-logarithmic scale we observe that all solutions demonstrate an exponential convergence as a function of polynomial order which is to be expected from this smooth solution. The LDG solution is 


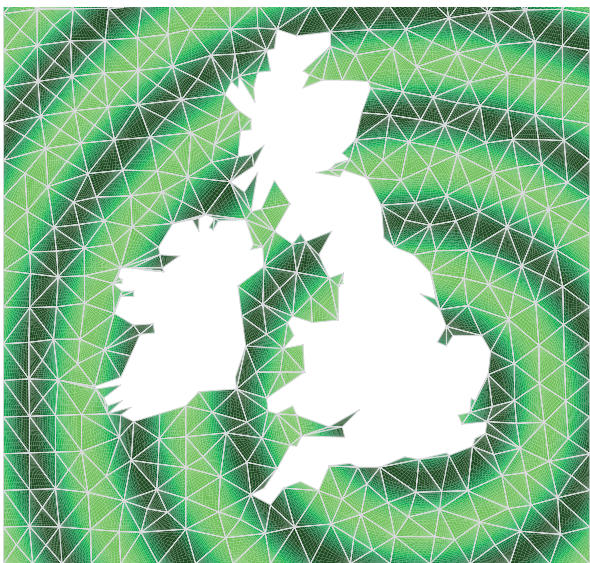

(a)

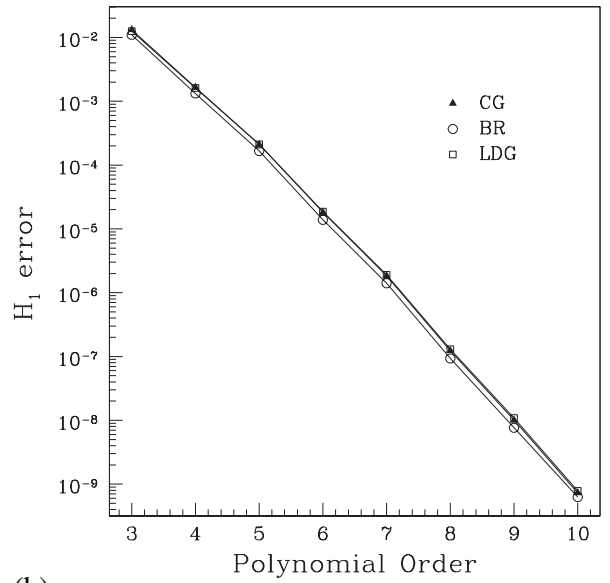

(b)

Figure 16. Helmholtz equation with an exact solution of the form $u\left(x_{1}, x_{2}\right)=\sin (1 / 4 \pi$ $\left.\sqrt{\left(x_{1}-a\right)^{2}+\left(x_{2}-b\right)^{2}}\right)$ on an unstructured triangular mesh (a). The error for the continuous Galerkin (CG) formulation and the DG formulation with Bassi-Rebay (BR) and LDG fluxes are shown in (b) as a function of polynomial order in the $H_{1}$ norm.

almost indistinguishable from the continuous Galerkin solution whilst the stabilized BassiRebay fluxes perform fractionally better. We note, however, that the LDG formulation contains more degrees of freedom than the continuous Galerkin formulation due to the duplication of element boundary degrees of freedom. Indeed the rank of the Schur complement system arising from the static condensation in the LDG formulation will be exactly double the rank of that corresponding to the continuous Galerkin formulation.

In the second test we consider a solution of the Laplace equation of the form $u(r, \theta)=$ $r^{2 / 3} \cos \left(\frac{2}{3}\left(\theta-\frac{\pi}{4}\right)\right)$ in an 'L'-shaped domain with Dirichlet boundary conditions. This domain is shown in Figure 17 where the origin $(r=0)$ was located at the internal corner of the domain. This solution satisfies Laplace's equation but has singular derivatives at the origin. Figures 17(a)-(c) show the pointwise evaluation of $\left|u_{x x}+u_{y y}\right|$ which should be zero and so acts as a measure of the error of the numerical solution. Figure 17(a) shows the solution when using a continuous Galerkin formulation whereas Figures 17(b) and (c) correspond to DG formulations using the Bassi-Rebay and normalized direction LDG fluxes, respectively. In all these figures the inset plot shows a close-up around the origin $r=0$.

In the close-up region of Figure 17(a) we observe that the continuous Galerkin solution primarily pollutes the regions immediately adjacent to the singular point $(r=0)$. Close inspection of this figure does however suggest a mild influence in the next layer of elements which most likely arises due to the larger stencil of the vertex modes. The influence of a larger stencil is far more evident in Figure 17(b) where we show the DG solution using Bassi-Rebay fluxes. Finally in Figure 17(c) we observe that the contours of $\left|u_{x x}+u_{y y}\right|$ are smooth outside of the region of elements immediately adjacent to the origin suggesting a smaller influence/pollution of the singularity in the solution domain.

To quantify this visual inspection of the solution we can consider the convergence of the solution as a function of polynomial order as shown in Figure 17(d). In this figure we show 

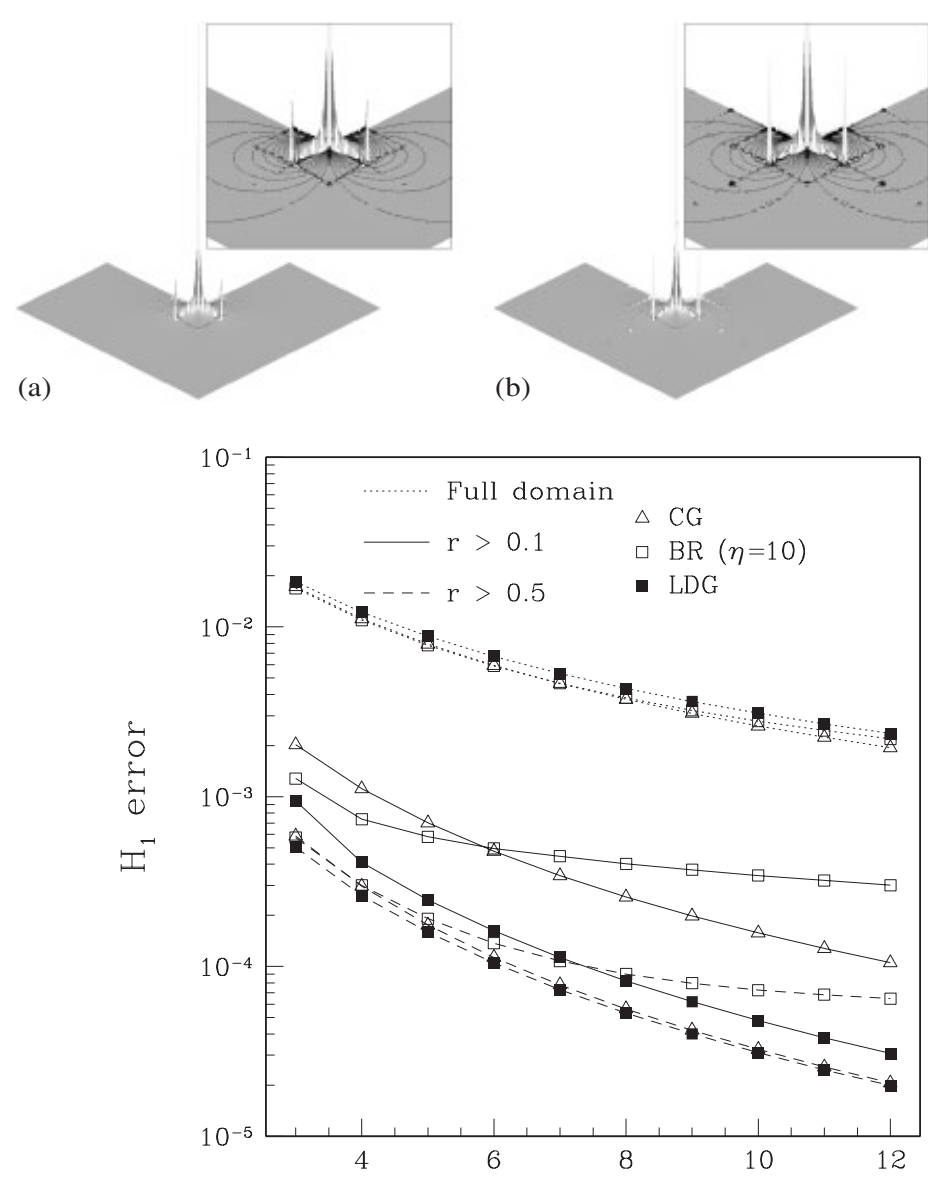

(d)

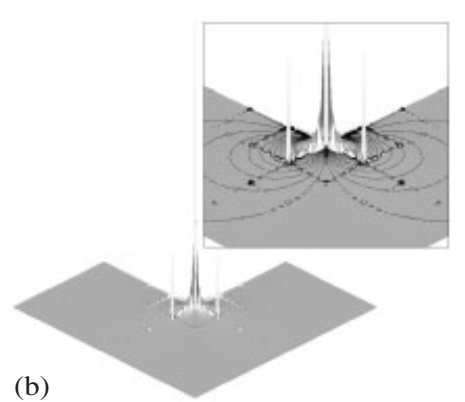

(c)

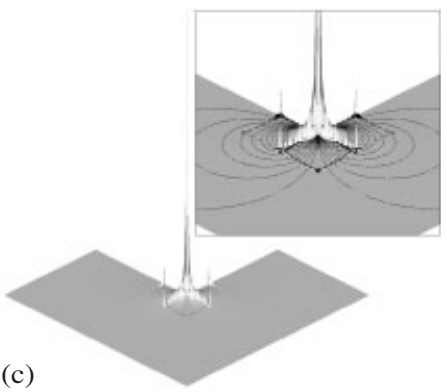

Figure 17. Solution to singular corner problem with solution $u(r, \theta)=r^{2 / 3} \cos (2 / 3(\theta-\pi / 4))$. Top plots show the pointwise evaluation of $\left|u_{x x}+u_{y y}\right|$ using: (a) continuous Galerkin formulation; (b) DG formulation with Bassi-Rebay fluxes and $\eta=10$; (c) DG formulation with LDG fluxes; and plot (d) shows the $H_{1}$ error of the three formulations in different regions of the solution domain indicated by plot (e).

the $H_{1}$ error evaluated using the elemental representation of the solution and its derivatives in different sub-regions. The dashed-dotted lines in this plot show the error of the three formulations over the whole solution domain. Within this region we observe that all schemes behave in a similar manner presumably being dominated by the error associated with the singularity at the origin. The solid lines show the convergence in a region which excludes the elements immediately neighbouring the singular point as shown by the diagonally shaded region in Figure 17(e) (calculated by excluding any elements with a vertex inside $r<0.1$ ). We observe that there is a marked difference between the convergence of the three formulations. The continuous Galerkin formulation starts with the highest errors at lower polynomial orders. 
The DG formulation with Bassi-Rebay fluxes performs slightly better at lower polynomial orders, but its convergence rate with polynomial order is slower than the continuous Galerkin formulation. At higher polynomial orders the error is highest for this scheme. The error of the DG formulation with normalized direction LDG fluxes demonstrates a notable improvement over the two previous schemes. At higher polynomial order of the error was almost three times lower than that of the continuous Galerkin formulation suggesting a reduction in the pollution error of this scheme. Finally, if we exclude the next layer of elements shown by the vertical shading in Figure 17(e) we obtain the convergence trends shown by the dashed lines. In this region the continuous Galerkin and DG scheme with normalized direction LDG fluxes perform similarly. The DG scheme with Bassi-Rebay fluxes converges similarly at lower polynomial orders but once again has a slow convergence rate and so a significant difference in the error appears at higher polynomial orders.

\section{ACKNOWLEDGEMENTS}

The authors would like to thank Prof David Darmofal of MIT, and his research group, for helpful and insightful comments on an early draft of this text.

The first author would like to acknowledge partial support of a global research award from the Royal Academy of Engineering.

The second author gratefully acknowledges the partial support of NSF Career Award NSFCCF0347791 and the computational support and resources provided by the Scientific Computing and Imaging Institute at the University of Utah.

\section{REFERENCES}

1. Bassi F, Rebay S. A high-order accurate discontinuous finite element method for the numerical solution of the compressible Navier-Stokes equations. Journal of Computational Physics 1997; 131:267-279.

2. Wheeler MF. An elliptic collocation-finite element method with interior penalties. SIAM Journal of Numerical Analysis 1978; 15:152-161.

3. Arnold DN. An interior penalty finite element method with discontinuous elements. SIAM Journal of Numerical Analysis 1978; 19:742-760.

4. Cockburn B, Shu C-W. The local discontinuous Galerkin for convection-diffusion systems. SIAM Journal on Numerical Analysis 1998 35:2440-2463.

5. Baumann CE, Oden JT. A discontinuous $h p$ finite element method for the Euler and Navier-Stokes problems. International Journal for Numerical Methods in Engineering 1999; 31:79-95.

6. Rivière B. Discontinuous Galerkin methods for solving the miscible displacement problem in porous media. Ph.D. Dissertation, The University of Texas, Austin, Texas, 2000.

7. Suli E, Schwab C, Houston P. hp-dgfem for partial differential equations with nonnegative characteristic form. In Discontinuous Galerkin Methods: Theory, Computation and Applications, Cockburn B, Karniadakis GEm, Shu C-W (eds). Springer: Berlin, 2000; 221-230.

8. Arnold DN, Brezzi F, Cockburn B, Marini D. Discontinuous Galerkin methods for elliptic problems. Discontinuous Galerkin Methods: Theory, Computation and Applications. Springer: Berlin, 2000; 135-146.

9. Arnold DN, Brezzi F, Cockburn B, Marini D. Unified analysis of discontinuous Galerkin methods for elliptic problems. SIAM Journal on Numerical Analysis 2002; 39:1749-1779.

10. Zhang M, Shu C-W. An analysis of three different formulations of the discontinuous Galerkin method for diffusion equations. Mathematical Models and Methods in Applied Sciences (MPAS) 2003; 13:395-413.

11. Atkins H, Shu C-W. Analysis of the discontinuous Galerkin method applied to the diffusion operator. 14th AIAA Computational Fluid Dynamics Conference, AIAA 99-3306, 1999.

12. Shu C-W. Different formulations of the discontinuous Galerkin method for the viscous terms. In Advances in Scientific Computing, Shi Z-C, Mu M, Xue W, Zou J (eds). Science Press: Beijing, 2001; 144-155.

13. Houston P, Schwab C, Suli E. Discontinuous hp-finite element methods for advection-diffusion-reaction problems. SIAM Journal on Numerical Analysis 2002; 39(6):2133-2163. 
14. Castillo P. Performance of discontinuous Galerkin methods for elliptic problems. SIAM Journal on Scientific Computing 2002; 24(2):524-547.

15. Kirby RM, Karniadakis GEm. Selecting the numerical flux in discontinuous Galerkin methods for diffusion problems. Journal on Scientific Computing 2005; 22-23(1-3):385-411.

16. Cockburn B, Karniadakis GE, Shu C-W. The development of discontinuous Galerkin methods. Discontinuous Galerkin Methods: Theory, Computation and Applications. Springer: Berlin, 2000; 135-146.

17. Cockburn B, Kanschat G, Perugia I, Schotzau D. Superconvergence of the local discontinuous Galerkin method for elliptic problems on Cartesian grids. SIAM Journal on Numerical Analysis 2001; 39(1):264-285.

18. Castillo P, Cockburn B, Perugia I, Schotzau D. An a priori error analysis of the local discontinuous Galerkin method for elliptic problems. SIAM Journal on Numerical Analysis 2000; 38(5):1676-1706.

19. Cockburn B, Kanschat G, Schotzau D, Schwab C. Local discontinuous Galerkin methods for the Stokes system. SIAM Journal on Numerical Analysis 2002; 40(1):319-343.

20. Cockburn B, Kanschat G, Schotzau D. The local discontinuous Galerkin method for the Oseen equations. Mathematics of Computation 2003; 73(246):569-593.

21. Zienkiewicz OC, Taylor R, Sherwin SJ, Peiro J. On the discontinuous Galerkin method. International Journal for Numerical Methods in Engineering 2003; 58(2):1119-1148.

22. Karniadakis GE, Sherwin SJ. Spectral/hp Element Methods in CFD. Oxford University Press: Oxford, 1999.

23. Brezzi F, Manzini G, Marini D, Pietra P, Russo A. Discontinuous finite elements for diffusion problems. Atti Convegno in onore di F. Brioschi (Milano 1997), Istituto Lombardo, Accademia di Scienze e Lettere, 1999; 197-217.

24. Baumann CE, Oden JT. A discontinuous $h p$ finite element method for convection-diffusion problems. Computer Methods in Applied Mechanics and Engineering 1999; 175:311-341.

25. Proriol J. Sur une famille de polynomes á deux variables orthogonaux dans un triangle. Comptes Rendus de l'Academie des Sciences Paris 1957; 257(2459).

26. Koornwinder T. Theory and Applications of Special Functions. Two-variable Analogues of the Classical Orthogonal Polynomials, Chapter. Academic Press: San Diego, CA, 1975.

27. Dubiner M. Spectral methods on triangles and other domains. SIAM Journal on Scientific Computing 1991; 6:345-390. 